\title{
Monitoring intracellular nanomolar calcium using fluorescence lifetime imaging
}

Kaiyu Zheng ${ }^{1}$, Thomas P. Jensen ${ }^{1}$, Dmitri A. Rusakov ${ }^{1}$

${ }^{1}$ UCL Institute of Neurology, University of London, London, UK

Correspondence should be addressed to: D.A.R. (d.rusakov@ucl.ac.uk) or K.Z. (k.zheng@ucl.ac.uk)

KEYWORDS Neuron, astroglia, synapse, plasticity, FLIM, fluorescence lifetime imaging, intracellular calcium, calcium imaging, gated intensity, nanomolar calcium, autofluorescence reduction, patch clamp, OGB-1, Oregon Green BAPTA1, calcium, two-photon imaging, brain slice, hippocampus, $\left[\mathrm{Ca}^{2+}\right]$.

EDITORIAL SUMMARY This protocol describes an improvedfluorescence lifetime imaging (FLIM) approach for nanomolar calcium imaging using the OGB-1 fluorescent calcium indicator (and similar indicators) in brain cells.

TWEET FLIM approach for imaging nanomolar calcium in brain cells.

COVER TEASER FLIM of nanomolar $\left[\mathrm{Ca}^{2+}\right]$ in brain cells 
Please indicate up to four primary research articles where the protocol has been used and/or developed:

1. Zheng et al. (2015) Time-resolved imaging reveals heterogeneous landscapes of nanomolar $\mathrm{Ca}^{2+}$ in neurons and astroglia. Neuron 88: 277-288.

2. Zheng et al. (2017) Nanoscale diffusion in the synaptic cleft and beyond measured with time-resolved fluorescence anisotropy imaging. Sci Reports 7:

10.1038/Srep42022

3. Jennings et al. (2017) Dopamine elevates and lowers astroglial $\mathrm{Ca}^{2+}$ through distinct pathways depending on local synaptic circuitry. Glia 65:447-459

4. Jensen et al. (2017) Monitoring single-synapse glutamate release and presynaptic calcium concentration in organised brain tissue. Cell Calcium 64: 102-108. 


\section{Abstract}

Nanomolar-range fluctuations of intracellular $\left[\mathrm{Ca}^{2+}\right]$ are critical for brain cell function but remain difficult to measure. We have advanced a microscopy technique to monitor intracellular $\left[\mathrm{Ca}^{2+}\right]$ in individual cells in acute brain slices (also applicable in vivo) using fluorescent lifetime imaging (FLIM) of the $\mathrm{Ca}^{2+}$-sensitive fluorescent indicator Oregon Green BAPTA1 (OGB-1). The OGB-1 fluorescence lifetime is sensitive to $\left[\mathrm{Ca}^{2+}\right]$ within the $10-500 \mathrm{nM}$ range but not to other factors such as viscosity, temperature, or $\mathrm{pH}$. This protocol describes the requirements, setup and calibration of the FLIM system required for OGB-1 imaging. We provide a step-bystep procedure for whole-cell OGB-1 loading and two-photon FLIM. We also describe how to analyse the obtained FLIM data using total photon count and gated intensity records, a ratiometric photon counting approach which provides a highly improved signal-to-noise ratio and greater sensitivity of absolute $\left[\mathrm{Ca}^{2+}\right]$ readout. We demonstrate our technique in nerve cells in situ, and it is adaptable to other cell types and fluorescent indicators. This protocol requires a basic understanding of FLIM and experience in single-cell electrophysiology and cell imaging. Setting up the FLIM system takes approximately two days, OGB-1 loading, imaging and data analysis takes two days. 


\section{INTRODUCTION}

Dynamic changes in intracellular $\mathrm{Ca}^{2+}$ concentration contribute to key signalling cascades in brain cells, including axonal release of neurotransmitters ${ }^{1}$, dendritic control of synaptic plasticity ${ }^{2,3}$, and intracellular communication in electrically passive astroglia ${ }^{4-7}$. In resting conditions, brain cells are believed to maintain their intracellular concentration of free $\mathrm{Ca}^{2+}\left(\left[\mathrm{Ca}^{2+}\right]\right)$ in the 20-200 nM range mainly through the continuous action of potent intracellular $\mathrm{Ca}^{2+}$ pumps and buffers. Sustaining this low $\mathrm{Ca}^{2+}$ concentration enables a wide dynamic range for a multitude of $\mathrm{Ca}^{2+}$-dependent molecular cascades. These cascades can be triggered by extracellular $\mathrm{Ca}^{2+}$ entering the cytoplasm via $\mathrm{Ca}^{2+}$ channels in the plasma membrane or from internal $\mathrm{Ca}^{2+}$ stores such as endoplasmic reticulum or mitochondria. Monitoring internal $\left[\mathrm{Ca}^{2+}\right]$ in neurons and glia thus remains one of the key gnostic windows into brain function, be it at the level of microscopic subcellular compartments ${ }^{8}$ or multi-cellular assemblies in the brain of living animals ${ }^{9,10}$.

Notwithstanding the progress in $\mathrm{Ca}^{2+}$ signal monitoring, the accurate measurements of intracellular $\left[\mathrm{Ca}^{2+}\right]$ in situ have remained technically challenging. Historically, highsensitivity $\left[\mathrm{Ca}^{2+}\right]$ monitoring in cultured cells has been performed by imaging ratiometric fluorescent $\mathrm{Ca}^{2+}$ indicators such as Fura $2{ }^{11,12}$. Although effective in tissue culture cells, this approach is hampered in organised brain tissue by the fact that light absorption and scattering strongly depend on the wavelength. This implies that the ratio of two or more chromatically separated signals, be it excitation or emission, can strongly depend on the recording depth. Therefore, $\left[\mathrm{Ca}^{2+}\right]$ monitoring in situ has commonly been carried out using fluorescence intensity-based measures 10,13-15. Whilst this approach has been highly successful in documenting large and rapid $\left[\mathrm{Ca}^{2+}\right]$ changes, its accuracy for detecting low $\left[\mathrm{Ca}^{2+}\right]$ levels (below $100 \mathrm{nM}$ ) is limited. However, resting (quasi steady state) $\left[\mathrm{Ca}^{2+}\right]$ has a direct influence on the presence of locally available $\mathrm{Ca}^{2+}$ buffers and hence the dynamics of physiological $\mathrm{Ca}^{2+16-20}$. 
In contrast to intensity measures, fluorescence lifetime imaging (FLIM) refers to the intrinsic stochastic decay of molecular fluorescence, which usually occurs on the nanosecond time scale. This decay is highly sensitive to the molecular microenvironment and does not depend on the dye concentration, the focus drift, laser power fluctuations, or light scattering. Various FLIM techniques have been developed to exploit this phenomenon for monitoring nanometer-scale molecular interactions in live cells and tissues ${ }^{21-23}$.

The fluorescence lifetime of one of the most common $\mathrm{Ca}^{2+}$ indicators, Oregon Green BAPTA-1 (OGB-1), is sensitive to $\left[\mathrm{Ca}^{2+}\right.$ ] in the nanomolar range ${ }^{24-26}$. Recently, we showed that the OGB-1 lifetime was also insensitive to physiological changes in $\mathrm{pH}$, $\left[\mathrm{Mg}^{2+}\right],\left[\mathrm{Zn}^{2+}\right]$, the concentration of a ubiquitous neural protein (actin), temperature, or micro-viscosity ${ }^{27}$ thus providing a sensitive and specific $\left[\mathrm{Ca}^{2+}\right]$ sensor in situ. By exploring this further, across various experimental scenarios, we were able to unveil and measure uneven landscapes of 'resting' $\left[\mathrm{Ca}^{2+}\right]$ in neurons and astroglia. In addition, we developed a specific photon counting procedure to enable rapid FLIM acquisition in situ ${ }^{27-29}$. The present protocol presents a systematic outline of the technical detail pertinent to this methodology (and immediately related applications), including a step-by-step description of the equipment calibration procedures and the gated-intensity analysis.

\section{Development of the protocol}

The FLIM technique described here is based on a time-correlated single-photon counting (TCSPC) instrument which has been widely used and well documented ${ }^{30}$. We have adapted the approach to image calcium concentrations in optically turbid tissues such as acute hippocampal slices and somatosensory cortex in vivo using two-photon excitation with a femtosecond infra-red laser pulses ${ }^{13,31,32}$. The analysis of FLIM data has long been the bottleneck in terms of the experimental throughput. We have therefore proposed to forego traditional multi-exponential fitting and replace it with a simpler and computationally economical approach based on a ratiometric 
method of normalised total count (NTC). The NTC technique we adapted proved highly efficient in mapping intracellular $\left[\mathrm{Ca}^{2+}\right]$ landscapes in neurons and astroglia in situ and in vivo, and it could be equally applicable in other cell types.

Similar to other FLIM protocols, the disadvantage of obtaining fluorescent decay data using TCSPC is a relatively long acquisition time per pixel, which is required to collect sufficient numbers of photon counts and thus to gather the acceptable fluorescence decay statistics. This approach could be suboptimal for measuring rapidly changing $\mathrm{Ca}^{2+}$ concentrations when compared to the more traditional intensity based methods. To overcome this, we have implemented a gated-intensity analysis which integrates the normalised fluorescence decay curve over a chosen time interval between the excitation onset and its tail end. This has significantly improved the signal-to-noise ratio of the fluorescence intensity measurements. We have shown that a modification of this method also enables separation of the target indicator signal (be it $\mathrm{Ca}^{2+}$ sensitive or not) from the concomitant auto-fluorescence ${ }^{27,33}$.

We have also adapted the two proposed approaches for FLIM data analysis, NTC and gated intensity, to the settings of intracellular $\left[\mathrm{Ca}^{2+}\right]$ measurements in brain cells in situ and in vivo using the lifetime of OGB-1 ${ }^{27-29}$.

\section{Applications}

The approach described here could be adjusted to other cell types and other fluorescence indicators that have similar fluorescence lifetime sensitivity to the molecular concentration of interest. One potentially important application of the NTC and gated intensity techniques is the optical separation of concomitant autofluorescence signals. In many cases, the autofluorescence signal is chromatically inseparable from common fluorophores, such as GFP. However, the lifetime of autofluorescence is often much shorter than that of exogenous fluorophores (which often display a $<2$ ns lifetime compared to $>3$ ns for GFP) ${ }^{34}$. In such cases, the gated intensity technique provides a straightforward solution, as described here. 
The NTC and gated intensity protocols presented here can be extended to various fluorescent microscopy setups and cell types once the fluorophore with the sensitive lifetime has been identified (see Experimental Design for details), calibrated, and tested for concomitant sensitivities (such as $\mathrm{pH}$, viscosity, temperature, etc). The present approach can therefore be applied to studies in physiological sciences, neurosciences, cell biology, biotechnology and other life science areas dealing with $\mathrm{Ca}^{2+}$-dependent cellular mechanisms acting in live tissue. Similarly, the protocol could be adapted, in principle, to fluorescent or phosphorescent lifetime sensors of any type.

\section{Comparison with other methods}

A conventional FLIM analysis of TCSPC data uses multiple exponential fittings, which provides information about indicator sub-populations with different lifetimes. Multi-exponential fitting is usually part of the acquisition software (e.g., PicoQuant) or the special analysis software (e.g., SPCImage from Becker \& Hickl). Despite the straightforward implementation of multi-exponential decay, establishing the analysis protocol itself can be time-consuming. In addition, the unambiguous interpretation of multiple fitting parameters is not always straight-forward. In many cases, different multi-exponential models need to be explored to achieve the optimal fit. For instance, in the case of OGB-1 at low $\left[\mathrm{Ca}^{2+}\right]$, triple-exponent fitting is required, whereas at higher calcium levels two exponents seem sufficient ${ }^{24}$. Thus, an algorithmic combination of exponential components (involving four or more free fitting parameters) needs to be established before being mapped to calibrated $\left[\mathrm{Ca}^{2+}\right]$. Once this is established, multi-exponential fitting has to be performed on a pixel by pixel basis, which can be a time-consuming and computational demanding task for large images (typically $256 \times 256$ to $512 \times 512$ pixels). When performing time-lapse imaging, typically involving hundreds of frames, this rapidly cumulates into an hour or more of computation for a single acquisition. This means that reanalysing such large datasets when the analysis protocol has to be adjusted or 
corrected becomes impractical. This is likely to be amongst the main reasons why large-scale FLIM mapping in biological research has been a rare occurrence.

Notwithstanding the important physical principles underlying exponential fitting, the information provided by it can be superfluous when the aim is to document and quantify overall changes in the fluorescence decay. Thus, to drastically cut down on the complexity of the analysis procedure, we opted for a much simpler approach of normalised (or ratiometric) total count ${ }^{27,29}$. The method provides a single value output, as opposed to, for instance, 6-8 parameters in the case of triple exponential fitting, and it thus maps seamlessly onto the respective $\left[\mathrm{Ca}^{2+}\right]$ values. In addition, there are no iterative computational procedures pertinent to the exponential fitting, which dramatically shortens the computational time required. The latter is a key prerequisite when analysing time-lapse imaging experiments (see Limitations).

The gated intensity approach has several advantages over the conventional fluorescent intensity measurements. Firstly, it widens the effective dynamic range and improves the signal-to-noise ratio. For instance, OGB-1 has a value of $F_{\min } / F_{\max }$ $\sim 4$ whereas Fluo-4 has a value close to 11 , owing to lower $F_{\min }$ (minimum fluorescence at lowest ion concentration) and higher $F_{\max }$ (maximum fluorescence at saturating ion concentration) ${ }^{13,35}$. The low $F_{\text {min }}$ implies that Fluo- 4 could be undetectable in small cellular structures at resting calcium levels. With the gated intensity method, OGB-1 can achieve $F_{\min } / F_{\max }>9$ (see below) while still providing robust measurements of resting $\left[\mathrm{Ca}^{2+}\right]^{27}$. Similarly evident is the improvement of the signal-to-noise ratio, which can unveil small $\left[\mathrm{Ca}^{2+}\right]$ changes undetectable with the intensity-based method ${ }^{27}$. Secondly, because the gated intensity method relies on the fluorescence lifetime features, it alleviates any concomitant influence of focus drift, light scattering, photobleaching or autofluorescence, by normalising gated intensity to its peak value (see Procedure).

Applying the gated intensity method to the removal of autofluorescence is highly beneficial for $\left[\mathrm{Ca}^{2+}\right]$ monitoring in some preparations and is particularly advantageous when applied to live human tissue samples in which intense, broad- 
spectrum autofluorescence is omnipresent ${ }^{33,36}$. In fixed tissues, the concomitant autofluorescence occurs mainly due to the polymerisation of fixatives, and it is usually dealt with by using chemical quenching ${ }^{37}$ or photobleaching ${ }^{38}$. These methods are however not applicable to live cells and tissues. ${ }^{36}$ The autofluorescence in human tissues is spectrally inseparable from that of any common 'green' $\mathrm{Ca}^{2+}$ indicator, such as OGB-1, Fluo-4, or GCaMP family (Table 1). Across different preparations, its concomitant effects range from a significantly reduced signal-to-noise ratio (and the dynamic range) to the virtually complete ablation of the functional indicator signal. The gated-intensity approach described in this Protocol appears the only practical way to remove the autofluorescence in such scenarios ${ }^{33}$. It also has an additional benefit of potentially increasing the dynamic range and signal-to-noise ratio for indicators with the condition-sensitive lifetime, such as OGB-1. Despite these advantages, its use in experimental research is rare.

\section{Limitations}

The main exemplar of our case study, hippocampal pyramidal cells, have been shown to maintain resting $\left[\mathrm{Ca}^{2+}\right]$ levels below $50 \mathrm{nM}^{27,39}$. OGB-1 is particularly suited for this range, as illustrated below. Other mammalian cells display a similar or higher resting $\left[\mathrm{Ca}^{2+}\right]$, typically up to $100-150 \mathrm{nM}$, such as brain astroglia ${ }^{27,28}$ or cardiac myocytes ${ }^{40}$. The $\mathrm{Ca}^{2+}$ indicator OGB-2, which has a similar lifetime sensitivity but higher $K_{d}$ (Table 1). Because the indicator's $K_{d}$ scales with the ion concentration that corresponds to a given ion bound-to-free ratio, OGB-2should be more suitable for the proportionally higher $\left[\mathrm{Ca}^{2+}\right]$ levels. The NTC technique should work well for the entire OGB family of fluorophores when applied to estimate and to map resting $\left[\mathrm{Ca}^{2+}\right]$ levels 41 . For applications that focus on relatively fast and wide-range concentration changes, OGB-1 could be suboptimal as it saturates at $\left[\mathrm{Ca}^{2+}\right] \sim 1 \mu \mathrm{M}$. Again, OGB-2 or OGB-5N appear better suited in such cases, for instance, when monitoring cardiac myocytes where $\left[\mathrm{Ca}^{2+}\right]$ could easily reach $>10-20 \mu \mathrm{M}^{40}$. 
Whilst the NTC method provides substantial computational and practical advantages over conventional multi-exponential fitting, its main limitation is that the probe calibration using NTC is equipment-dependent. In a conventional multipleexponential fitting method, the measured or estimated finite instrumental response (FIR) will be deconvolved from the data. Therefore, the data acquired by different optical systems (e.g. with different detectors or laser sources) should not, in theory, significantly affect the fitting results. With the NTC method, the FIR is assumed to be constant, which is the case when the imaging system remains unchanged. However, the indicator sensitivity will have to be recalibrated whenever system components that affect FIR (such as detectors or lasers) are changed. In practice, however, changing a (two-photon excitation) FLIM microscopy system is a very infrequent event.

Similar to other FLIM techniques, one general limitation of the NTC analysis is the trade-off between maximising photon count rate towards the decay tail (which improves sensitivity) and maximising image acquisition rate. In the case of OGB-1, reducing the integration window from 9 ns to $5 \mathrm{~ns}$ avoids low photon numbers at the tail but also reduces method sensitivity. Again, an optimal trade-off could be achieved during empirical exploration.

In contrast, the gated-intensity approach is much less dependent on the photon count rate. This is because here we deal with a large difference between large integration intervals, rather than with a small difference between large integration intervals in the NTC method. Thus, the gated-intensity method is far less sensitive to photon count rates, for instance, when measuring low $\left[\mathrm{Ca}^{2+}\right]$. When this method aims to separate the autofluorescence signal, the only limitation is that the observed autofluorescence lifetime must be significantly shorter (ideally half or less) than the functional lifetime of the target indicator. Otherwise, conventional multiple exponential fittings (often enabled in the unit-bundled software) could be used to achieve the same goal, albeit at a much higher computational cost. 


\section{Experimental Design}

Choosing a suitable fluorescent indicator: The first key step in successfully using FLIM combined with NTC and gated intensity is to choose a suitable fluorescent indicator. In order for both the NTC and gated intensity method to work well, robust sensitivity of the fluorescent lifetime decay to a stimulus (e.g., ion concentration change) is desirable. There is a body of FLIM measurements obtained to date that enable scientists to choose their ideal fluorescence indicator (see examples in Table 1). Other technical requirements for an 'ideal' candidate fluorophore are: (a) reasonably strong emission in stimulus-free conditions, (b) a fluorescent lifetime $I_{\text {free }}$ larger than the finite instrumental response (FIR) of the TCSPC system in stimulusfree conditions, and (c) a rapid anisotropy decay rate, which scales with molecular diffusivity and should typically be $<<1 \mathrm{~ns}$ in the cytosol ${ }^{42}$ (i.e. very large fluorophores might display depolarisation which is too slow in the target medium). For instance, the $\mathrm{Na}^{+}$-sensitive indicator SBFI has a $I_{\text {free }}$ which is very close to FIR of our system 43. The difference in lifetime between free and bound states ( $\left.\tau_{\text {free }}-T_{\text {bound }}\right)$ has to be as large as possible. The fluorescent indicator used in the present protocol, OGB-1, has a ratio of $\tau_{\text {bound }} / T_{\text {tree }}$ around 5.5 . Other possible candidates from Table 1 are OGB-2, Calcium Green and Magnesium Green. Others, such as Sodium Green, would be difficult to implement using the approach described in this protocol because their fluorescence lifetime appears largely insensitive to ion concentrations 43.

Overview of essential FLIM equipment: To measure fluorescence lifetime, the essential equipment comprises an excitation source (laser), a laser-scanning microscope, good quality optical filters and dichroics, FLIM detectors, a time correlated single photon counting (TCSPC) electronics system including recording and analysis software. The TCSPC system could be commercially supplied as a whole system or available as a turn-key microscope upgrade from several major suppliers, such as Becker \& Hickl and Picoquant . Specifications of individual components depend on the fluorescent sensors to be used, the biological system to 
be studied and the budget available. In the sections below we provide some general guidelines for choosing the appropriate equipment.

Excitation Laser source: Any pulsed laser with a short pulse width of 200 ps or less and a repetition rate between $20-100 \mathrm{MHz}$ should work well with the common TCSPC systems. The pulse repetition rate will set the duty cycle period and thereby limits the fluorescence lifetime to be measured. For instance, an $80 \mathrm{MHz}$ laser will have a duty cycle of $12.5 \mathrm{~ns}$, which is ideal for most fluorescence lifetime decays (see examples in Table 1). To image deep into turbid samples such as acute brain slices or in vivo tissue, two-photon excitation with infra-red light is essential. Therefore, a pulsed infrared laser is preferable. In our case, we use a tuneable Newport-Spectraphysics Ti:Sapphire MaiTai laser, which pulses at $80 \mathrm{MHz}$, with a pulse width of $\sim 220 \mathrm{fs}$ and wavelength set at $800 \mathrm{~nm}$.

Commercial TCSPC setups: Most common laser scanning microscopes from Zeiss (e.g., LSM880), Leica (e.g., Leica SP5), Olympus (e.g., FV1000), or Femtonics (e.g., Femto3D-RC or Femto2D-FLIM) can have TCSPC components added by the supplier (Fig. 1). These components are user-friendly and require minimal prior knowledge on operating TCSPC systems. TCSPC component suppliers, such as Becker \& Hickl and PicoQuant, will provide expertise and advice for setting it up.

Custom-built TCSPC set up: It is also feasible to custom build TCSPCs and connect them the laser scanner system, which can be done when older systems (e.g. Biorad Radiance 2000) are being used or where budget constraints exist. However, this requires more extensive knowledge of electronics, optics and the working principles of TCSPC. In order to reconstruct images using the photon counting information accumulated in a TCSPC system, the appropriate pixel, line and frame clock signals from the scanning system will have to be passed onto the system. In order to achieve this, scan clocks containing pixel, line and frame from the output of the laser scanning unit can be connected to the TCPSC system directly. It is important to make sure the edge direction of the clocks is set appropriately in the acquisition software, since the clock profile may be different than 
the TCSPC unit expects. For example, the Biorad Radiance 2000 system has an edge trigger for each pixel instead of a level trigger, in which case a fast delay pulse generator (e.g. BNC505, Berkeley Nucleonics Corp, San Rafael, CA) is needed to correct the signal and to feed it forward. External triggering signal is also desirable, to enable synchronisation with other equipment units. Becker \& Hickl provide a comprehensive TCSPC system overview, setup and troubleshooting in their handbook ${ }^{44}$.

Detectors: Basic requirements for optical detectors are the same as for any fluorescence microscope setup with regards to the wavelength sensitivity and quantum yield. However, due to the TCSPC temporal discrimination technique, a high detector voltage gain, short output pulse width ( $<<1 \mathrm{~ns}$ ), good electronic noise shielding and low dark current are essential. In depth analysis is available in the Becker \& Hickl handbook ${ }^{44}$. Detectors used in our example are PMC-100 from Becker \& Hickl and PMA-40 from PicoQuant. Make sure that the output of the detector is directly connected to the photon channels in the TCSPC unit.

Optical filters and dichroics: Because of the high sensitivity of the detector, it is important to use high quality dichroics and filters to optically shield detectors from scattered infrared laser light and to chromatically separate individual wavelength channels. Semrock dichroics and filters are used in our case. The $735 \mathrm{~nm}$ edge BrightLine multiphoton single-edge dichroic beam splitter (FF735-Di02-25×36) is used together with $720 \mathrm{~nm}$ blocking edge BrightLine multiphoton short-pass emission filter (FF01-720/SP-25). This combination is essential for a two-photon excitation imaging system. Additional filters are optional for the bandpass selection of OGB-1 signals, and only needed when secondary morphological fluorophores (e.g. Alexa Fluor 594 or SR101) are used. In which case, a $546 \mathrm{~nm}$ blocking edge BrightLine short-pass filter (FF01-546/SP-25) or 560 nm edge BrightLine singleedge dichroic beam splitter (FF560-Di01-25×36) would block or separate any signal coming from OGB-1 and longer-wavelength fluorophores. 
Optical arrangement: Split the output fluorescence from the microscope, as close to the back aperture of the objective as possible, using excitation dichroics (D1 in Fig. 1). The coupling can be free path or via an optical fibre. Due to the sensitivity of the TCSPC unit, robust light shielding is vital to reduce background count.

Time correlated single photon counting (TCSPC): Two major suppliers of TCSPC systems are Becker \& Hickl (http://www.becker-hickl.de/tcspc.htm) and PicoQuant (https://www.picoquant.com/products/category/tcspc-and-time-taggingmodules) who provide a wide selections of components and systems. For simple single- or dual-channel (wavelength or polarisation) recordings shown in the present Protocol, PicoHarp300 from PicoQuant and SPC830 from Becker \& Hickl were used.

Pre-amplifier module: A synchronisation signal from the laser excitation source is needed for discrimination between the photon arrival time and the excitation pulse time, in order to establish fluorescent lifetime. In order to do so, the $80 \mathrm{MHz}$ electronic triggering output has to be routed from the laser source into the TCSPC unit. Use an oscilloscope to make sure the electronic signals from the laser head are of sufficient quality, in accordance with the TCSPC specification. If an oscilloscope is not readily available, acquisition software will provide a SYNC count which should approximate the quoted laser pulse repetition rate. The laser head BNC output can often be noisy, particularly at non-optimum wavelength in a tuneable laser system. This can be seen via the SYNC count which will fluctuate or report inaccurate values. In such cases, a pre-amplifier module can be added to clean up and boost electronic output from the laser head to provide an accurate sync rate. In this protocol, PAM102 from PicoQuant and HFAC-26 from Becker \& Hickl were used with their respective TCSPC systems. Alternatively an optical pulse picker can be coupled to a beam splitter (BS1 in Fig. 1).

Optical pulse picker (optional): Synchronisation signal can also be better provided by using an optical pulse picker and a $5 / 95 \%$ or $1 / 99 \%$ beam splitter set at the output of the laser source (Fig. 1). Although this is more expensive, this option is preferred over the electronic synchronisation output from the laser head because it provides cleaner signals at all output wavelengths. 
Signal router: In a dual-channel (polarisation) setup ${ }^{42}$, a signal router is also needed to differentiate photons arriving to the two different detectors. We used Becker \& Hickl HRT-41 coupled with two of Becker \& Hickl's PMC-100 detectors.

Acquisition and analysis computing environment: A good quality, workstationgrade PC should be used to ensure robust and reliable experiment performance. Various systems have their own hardware connectivity specifications, such as the number of $\mathrm{PCI}$ slots and USB ports. The general rules to follow are: to maximise RAM (16GB or more is preferable for time-lapsed imaging, 4GB minimum for static $2 \mathrm{D}$ images), at least quad core $\mathrm{CPU}, 64$-bit operating system, fast storage medium (e.g. SSD HD). The acquisition software packages that accompany TCSPC systems are simple to operate; the experimenter only needs to optimise few simple settings to enable various modes of image acquisition. It is important to note that the analogue-to-digital converter (ADC) resolution for nanosecond delay time should typically be set to 256 or lower. In our experience, any higher ADC resolution provides no additional benefits for our analysis method while prolonging the acquisition time required for the same measurement. The NTC and gated intensity analysis procedures are simple by themselves, but when applied to large 4D dataset it is best done by using automated software, either by adopting an existing package (e.g. SymPhoTime64 from PicoQuant, which has scripting capacity, available at https://www.picoquant.com/products/category/software) or by writing a custom code (in our case it was FIMAS in MATLAB, available at https://github.com/zhengkaiyu/FIMAS).

System calibration: Once all the components have been connected and set up, and prior to the measurements, it is important to check the detector dark count rate. This is the count rate when the system is made fully light proof (blacked out) and its excitation light source is switched off. A typical dark count rate is $200-900 \mathrm{cps}$. Anything higher than that indicates either detector failure or insufficient light shielding. One cannot stress strongly enough that even the smallest gap for light escape can increase the dark count rate significantly. Therefore it is vital to check for light shielding before checking any other hardware units. Next, turn on the 
excitation light source and scanning, with no samples, to make sure the light source is well blocked inside the optical system by filters and dichroics.

Finally, tune and check the finite instrumental response (FIR) of the system. Typically, a urea crystal can be used as a second harmonics generator (SHG) for this purpose. Thin $\mathrm{Al}_{2} \mathrm{O}_{3}$ surfaces (e.g. kitchen foil surfaces) generated surface plasmon can also be used. Fluorophores with an extremely short lifetime (naturally or quenchend) compared to the detector response time may also be used ${ }^{30,45}$ (for FIR protocol procedures see a detailed practical guide by Becker \& Hickl at http://www.becker-hickl.com/pdf/irf-mp04.pdf). Microscope scanning with one of these samples under the objective should produce a sharp FIR pulse displayed in the acquisition software. Adjust the cable length for a sync signal and detector signal(s) to place the FIR peak ideally between $0.5 \mathrm{~ns}$ and $1 \mathrm{~ns}$. If there are periodic ripples in the FIR after the main peak, one should check for optical reflective surfaces in the optical pathways. Use components with anti-reflection coating if necessary. The Becker \& Hickl handbook ${ }^{44}$ provides an exceptionally detailed setup instruction for wide-ranging types of imaging systems. It is worthwhile to consult it even for TCSPC systems other than manufactured and installed by Becker \& Hickl.

Once the system has been optimised for TCSPC FLIM measurements, carry out careful calibration of the chosen fluorophore to the stimulus (such as calcium) for NTC method (see Procedure: Calibration): the accuracy of any subsequent measurements will critically depend on this (Steps 1-49 in Supplementary Fig. 1). In the course of calibration, it is vital to establish that the fluorophore lifetime is insensitive to other common environmental factors. A particularly ubiquitous factor is $\mathrm{pH}$, in response to which most fluorescent indicators change their properties ${ }^{46}$. The task here is to establish whether the $\mathrm{pH}$ sensitivity is within the physiological $\mathrm{pH}$ range under study. Another important factor is other ions of the same valence. Thus, calcium indicators could also be sensitive to other divalent cations, such as $\mathrm{Mg}^{2+}, \mathrm{Cs}^{2+}, \mathrm{Zn}^{2+}, \mathrm{Pb}^{2+}$, because of the similarities in the electron shell configuration which underpins the process of molecular binding ${ }^{47}$. It is imperative to check 
indicator lifetime sensitivity to the physiological relevant ranges of ion concentrations as the indicator is likely to produce biased readout if there are biologically-triggered changes of these ions during an experiment. Other factors such as temperature, viscosity, or the presence of ubiquitous proteins (e.g., albumins or actins) may also interact with large fluorophore molecules. Overall, the aim of the calibration process is to establish that the measurement readout is specifically sensitive to the stimulus of interest (for instance, $\left[\mathrm{Ca}^{2+}\right]$ ) and is not significantly biased by the likely concomitant effects within the physiological range of the system under observation. In our case, we used a standard internal solution for whole-cell electrophysiology as the base solution for all calibrations (Supplementary Fig. 1). Note that the sensitivity of fluorophores to various environmental factors may affect not only FLIM measurements but also the conventional intensity readout, although the underlying physical mechanisms could differ.

Generally, for the method to work well, introducing the chosen fluorophore to the sample should maximise photon count. In our experience, loading cells via electroporation, bulk AM dye application, or using genetically engineered fluorescent indicators currently appears less preferable compared to whole-cell loading, at least in the present context. This is mainly because whole-cell loading achieves high concentrations and even distribution of the indicator across the cytoplasm while providing valuable electrophysiological data to monitor cell condition and function. A continued supply of the indicator through the patch-pipette also makes it insensitive to photobleaching. It is however noteworthy that achieving patch clamp of principal neurons in older rats has traditionally been difficult, partly because of the tougher (more glia enriched) tissue conditions. In contrast, it has been possible to routinely patch astroglia and fill them with fluorescence indicators in relatively old rodents ${ }^{48}$ (see https://www.brainslicemethods.com/ for technical detail).

Experimental considerations: The imaging protocol itself has to be considered carefully, and decided upon at the experiment design stage. Given the inherent FLIM prerequisite of having the minimal necessary photon count rate, imaging small volumes such as small axonal boutons or dendritic spines of nerve cells poses a 
challenge. For steady state measurements in a small region of interest (ROI; e.g., axonal boutons or dendritic spines of nerve cells), long acquisition periods (multiple duty cycles) will be required to register enough photons to build decay statistics. A long exposure however can be prone to stage drift or focus fluctuations which may render the results invalid. One should however resist the temptation to increase laser power in order to reduce exposure time, because high laser excitation tends to elevate $\left[\mathrm{Ca}^{2+}\right]_{i}$ and detriment to cell health. $\mathrm{A}$ trade-off is therefore required between long acquisition time (to minimise data binning at the analysis stage) and minimal laser exposure (to minimise its harmful effects and increase acquisition speed). Often it is more efficient to form a mosaic of smaller ROls to cover a larger area. For measurements aimed at detecting rapid changes, spatial resolution has to be sacrificed - either by imaging smaller areas, with limited pixel numbers $(<50)$, or by using the line scan mode (straight or spiral). The latter provides two key advantages: increased temporal resolution and an improved photon count rate hence reduced acquisition time.

The NTC method has only one critical FLIM parameter for calculation, namely the end of the integration interval (the start is at the emission peak). This is limited by the laser pulse repetition rate (duty cycle) and the position of the peak which can be shifted. Depending on the actual fluorescent decay profile, this interval can be adjusted to individual experimental conditions. It is however vital to remember that the calibration function has to be analysed using the same integration interval throughout. This does not affect the way the calibration measurements were acquired but only the subsequent analysis of it.

The gated intensity method also has only one critical parameter for its calculation, namely the start of the integration interval from the tail. For the fluorescence lifetime decaying exponentially, the main contrast usually comes from the tail end of the decay. Therefore, in theory, the closer the interval onset is to the tail end the better the contrast. At the same time, however, because of lower photon counts towards the tail, placing the interval onset towards the very end will increase noise in the 
resulting trace. To achieve an optimal trade-off between the contrast and the noise it is recommended to establish the interval onset empirically.

\section{MATERIALS}

\section{REAGENTS}

- Mouse or rat Brain slices (see Reagent Setup). CAUTION: Any experiments involving live animals must conform to relevant Institutional and National regulations. The procedures described in this protocol were carried out under the Home Office project licence PPL 70/7524, in accord with the UK Animals (Scientific Procedures) Act 1986....

- Double distilled water (Sigma W3500) CRITICAL: Water quality is important for calibration solutions; use an unopened bottle

- Methanesulphonic acid (Sigma 471356); CRITICAL do not use the salt form, such as Potassium Methanesulphonate, to avoid impurities

- Potassium Hydroxide (Fisher J/6630/PB15) CRITICAL: Reagent purity is essential for calibration solutions; use sealed containers

- $\quad$ HEPES, tirate (Sigma H3375)

- $\quad$ Tris-Phosphocreatine (Sigma P1937)

- $\quad$ di-Sodium ATP (Sigma A2383)

- $\quad$ di-Sodium GTP (Sigma G8877)

- Magnesium Chloride (Sigma M2670) CRITICAL: this compound normally contains some calcium impurities, use analytical grade where possible

- $\quad$ Oregon Green 488 BAPTA-1, hexapotassium salt (Invitrogen 0-6806)

- $\quad$ BAPTA (Sigma A4926)

- $\quad$ BAPTA tetrapotassium salt (Invitrogen B1204) 
- $\quad$ Potassium Chloride (Sigma P9333)

- $\quad$ Calcium Chloride (Sigma C3881)

\section{EQUIPMENT}

CRITICAL: Different TCSPC systems have their own particular arrangements. However, principal components and the overall optical and electronics arrangements are the same for all systems, as indicated by the schematic in Fig. 1 and listed below.

- $\quad$ Excitation source: femtosecond Ti:Sapphire HP laser pulser Mai-Tai (SpectraPhysics-Newport)

- $\quad$ Beam expander (Thorlabs GBE05-B); serves to reduce possible damage to filters, dichroics and other optical components

- $\quad$ Optical shutter and controller (Thorlabs SH05 and SC10); CRITICAL set up the shutter control unit to minimise sample laser exposure

- $\quad$ Periscope assembly (P1 in Fig. 1, Thorlabs RS99 and UM10-AG); CRITICAL use low Group Delay Dispersion mirrors

- Variable ND filter (Thorlabs NDC-100C-4M); CRITICAL use Pockels cell or a polarisation based retarder to modulate high power laser source

- $\quad$ Laser scanning microscope (Biorad Radiance 2000, Olympus FV1000, or Femto2D-FLIM).

- $\quad$ Delay pulse generator (Berkeley Nucleonics model 505)

- $\quad$ Optical filter and dichroic set (FF735-Di02-25x36, FF01-720/SP-25, FF01546/SP-25, FF560-Di01-25x36); no magic-angle polarisers were used in our imaging settings to correct for anisotropy effects; CRITICAL: Check the incident light direction on the filter mount and dichroics; always check correct filters have been used on the day.

- $\quad$ Detectors (Becker \& Hickl PMC-100 or PicoQuant PMA-40); CRITICAL always shield detectors from background light by working in optically well isolated cages and rooms 
- $\quad$ TCSPC main electronics (Becker \& Hickl SPC830 or PicoQuant PicoHarp 300); CRITICAL handle with anti-static devices to minimise damage risk to sensitive electronics

- $\quad$ Pre-amplifier module (Becker \& Hickl HFAC-26 or PicoQuant PAM102);

CRITICAL handle with anti-static devices to minimise damage risk to sensitive electronics

- $\quad$ Signal router (Becker \& Hickl HRT-41); CRITICAL handle with anti-static devices to minimise damage risk to sensitive electronics

- $\quad$ Computer hardware (HP/Acer Workstation or similar); CRITICAL make sure use high end workstation grade PC with sufficient ports and RAM are present.

- $\quad$ Computer software packages SPCM, DCC (Becker \& Hickl, available at http://www.becker-hickl.de/software.htm), SPClmage, or SymPhotime 64 (PicoQuant, available at https://www.picoquant.com/products/category/software). Otherwise, custom software FIMAS current version available at https://github.com/zhengkaiyu/FIMAS under MATLAB (2015b or later, 64bit OS)

- $\quad$ Microscope stage (e.g., shifting table XY- shifting table 380FM-U, Luigs \& Neumann) with a slice recording chamber (e.g., Luigs \& Neumann, Cat No 200-100 500 0150).

\section{Electrophysiology equipment}

- $\quad$ An electrophysiology setup for submerged brain slices equipped with 40-60x DIC IR visualization, a patch clamp amplifier (e.g., MultiClamp 700B, Axon Instruments) and microelectrode positioners (e.g., Mini 25 manipulator block $X Y Z$, Luigs \& Neumann). An example of the working setup coupled with the two-photon excitation and two-photon uncaging system was described earlier 49 
- $\quad$ Micro-pipettes for whole-cell recording and dialysis are pulled from borosilicate glass (GF150F-3, Warner Instruments) using a Sutter Instuments P-97 or Zeitz Instruments DMZ Universal electrode puller.

\section{Brain slicing equipment}

- $\quad$ A vibrating microtome, Leica VT1200S or a Campden Instruments 7000 smz was used for preparation here. Other models are available but the ability to correct for vertical blade oscillations is preferable for obtaining best results.

\section{REAGENTS SETUP}

Brain slices Prepare acute, 350- $\mu \mathrm{m}$-thick transverse slices from isolated rat or mouse hippocampi using an appropriate standard technique, optimised to preserve the main hippocampal circuitry ${ }^{50-52}$. In our case, acute slices are cut in an ice-cold slicing solution (see Reagent Setup) and then stored in the same solution at $34^{\circ} \mathrm{C}$ for 15 min. CAUTION: Any experiments involving live mice/rat must conform to relevant Institutional and National regulations. The procedures described in this protocol were carried out under the Home Office project licence PPL 70/7524, in accord with the UK Animals (Scientific Procedures) Act 1986. .

\section{Stock solutions}

CRITICAL Make all stock solutions for $1 \mathrm{M} \mathrm{KOH}, 100 \mathrm{mM} \mathrm{KOH}, 1 \mathrm{M} \mathrm{MgCl}_{2}, 1 \mathrm{M}$ $\mathrm{CaCl}_{2}$ and $10 \mathrm{mM} \mathrm{OGB}-1$ fresh on the day. Use only recently purchased unopened containers for solutions containing calcium buffers and chemicals, including OGB-1, BAPTA, BAPTA salt, and di-Sodium ATP.

Extracellular solutions Slicing solution contains (in $\mathrm{mM}$ ): $\mathrm{NaCl} 60$, sucrose 105, $\mathrm{NaHCO}_{3} 26, \mathrm{KCl} 2.5, \mathrm{NaH}_{2} \mathrm{PO}_{4} 1.25, \mathrm{MgCl}_{2} 7, \mathrm{CaCl}_{2}$ 0.5, glucose 11, Ascorbic acid 1.3 and Sodium pyruvate 3 (osmolarity 300-310 mOsM). Recording solution 
contains (in mM): $\mathrm{NaCl} 125, \mathrm{NaHCO}_{3} 26, \mathrm{KCl}$ 2.5, $\mathrm{NaH}_{2} \mathrm{PO}_{4}$ 1.25, $\mathrm{MgSO}_{4}$ 1.3, $\mathrm{CaCl}_{2}$ 2 and glucose 16 (osmolarity 300-305 mOsm). Extracellular solutions must be continuously bubbled with $95 \% \mathrm{O}_{2} / 5 \% \mathrm{CO}_{2}$. Slices are allowed to rest for at least 60 min before recordings started.

Intracellular solution Mix $30 \mathrm{~mL}$ of double distilled water with $0.438 \mathrm{~mL}$ Methanesulphonic acid (final concentration: $135 \mathrm{mM}$ ). Add $\mathrm{KOH}$ initially from the $1 \mathrm{M}$ stock to increase the $\mathrm{pH}$ to 6-6.5 and switch over to the $100 \mathrm{mM} \mathrm{KOH}$ stock to increase the $\mathrm{pH}$ to 7.0. Add the following compounds in sequence (final concentration): 119mg HEPES (10 mM), 227mg Tris-Phosphocreatine (10 mM), $110 \mathrm{mg}$ di-Sodium ATP $(4 \mathrm{mM}), 11 \mathrm{mg}$ di-Sodium GTP $(0.4 \mathrm{mM})$. Add $200 \mu \mathrm{l}$ of $1 \mathrm{M}$ $\mathrm{MgCl}_{2}$ (4 mM). Adjust the $\mathrm{pH}$ again carefully to 7.2 using the $100 \mathrm{mM} \mathrm{KOH}$ stock solution. Add double distilled water to get 295-310 mOsm osmolarity, which should produce a desired final solution volume of $50 \mathrm{~mL}$. $100 \mu \mathrm{L}$ aliquots can be stored at $20^{\circ} \mathrm{C}$ for up to 3 months.

CRITICAL Add $200 \mu \mathrm{L}$ OGB-1 into thawed aliquot before the experiment from a fresh $10 \mathrm{mM}$ stock to make up the final concentration (e.g. $200 \mu \mathrm{M}$ ).

\section{Calibration-base solutions (High Calcium Solution HS and Low Calcium}

Solution LS) These two solutions (detailed in Table 2) are used to prepare the full range of calibration solutions for the required range of $\left[\mathrm{Ca}^{2+}\right]$.

Prepare twice the amount of Intracellular solution as described above but without the final step of adding $\mathrm{dd}_{2} \mathrm{O}$ to make up the final volume (volume $\sim 60 \mathrm{~mL}$ ). Divide the solution into two equal amounts into plastic beakers labelled HS and LS respectively (Supplementary Fig. 1). Add $119 \mathrm{mg}$ and $238 \mathrm{mg}$ of BAPTA to the HS (5 mM) and LS (10 mM) solutions respectively. Add $157 \mathrm{mg}$ BAPTA tetrapotassium salt to the HS solution ( $5 \mathrm{mM}$ ). Add $1 \mathrm{M} \mathrm{KOH}$ stock solution carefully to help dissolution of large quantity of BAPTA and to neutralise the $\mathrm{pH}$ of both solutions to 7.0 . Add $75 \mathrm{mg} \mathrm{KCl}$ to the LS solution (20 mM). Add $0.5 \mathrm{~mL}$ of $1 \mathrm{M} \mathrm{CaCl}_{2}$ into the $\mathrm{HS}$ solution $(10 \mathrm{mM})$. Carefully add $100 \mathrm{mM} \mathrm{KOH}$ stock solution into both $\mathrm{HS}$ and LS solutions to allow $\mathrm{pH}$ 
to reach 7.2 in both. Add $d_{d H_{2}} \mathrm{O}$ to both solutions so that the final volumes are 50 $\mathrm{mL}$ each. Because of the additional $20 \mathrm{mM} \mathrm{K}+$ in the calibration base solutions and extra $\mathrm{KOH}$ needed to neutralise BAPTA acid, the osmolarity of the calibration base solutions will be around $360 \mathrm{mOs}$, which is slightly higher than the usual intracellular solution (295-310 mOs). To calibrate against other environmental factors, a similar approach can be used to create high and low base solutions.

CRITICAL Because the calibration process is critical to the accuracy of any subsequent analysis using NTC method, the calcium base solutions must be made fresh. Once made, keep HS and LS solutions at room temperature $\left(20-25^{\circ} \mathrm{C}\right)$ until use.

\section{Calibration solutions}

The detailed contents of the individual calibration solutions and the procedure to obtain them are described in Table 3 and Supplementary Fig. 1. All calibration solutions should be prepared fresh and stored at room temperature until use.

\section{PROCEDURE}

\section{Calibration}

For calibration purposes, fluorescence lifetime measurements are carried out in each calibration solution with the acquisition (photon collection) time sufficient to obtain noiseless decay kinetics (Fig. 2a). The normalised photon count (NTC) values are calculated as the ratio between the area-under-the-curve (onset at the peak; interval, $9 \mathrm{~ns}$ ) and the peak value (Fig. 2a). Once plotted against the $\left[\mathrm{Ca}^{2+}\right]$ values, the dependence is well approximated with a logistic function (Fig. 2b, Supplementary Fig. 1) which is used as a calibration reference for in situ measurements.

\section{[Troubleshooting]}




\section{Acute slice preparation $\bullet$ Timing: 100-110 minutes}

1 Prepare acute, 350- $\mu \mathrm{m}$-thick transverse slices from isolated rat or mouse hippocampi using an established technique, optimised to preserve the main hippocampal circuitry ${ }^{50-52}$. Here, acute slices are cut on a Leica VT1200S or a Campden Instruments 7000smz in an ice-cold slicing solution (see Reagent Setup) and then stored in the same solution at $33-34^{\circ} \mathrm{C}$ for $15 \mathrm{~min}$. CRITICAL STEP: Both sucrose slicing solution and extracellular solution must be continuously bubbled with $95 \% \mathrm{O}_{2} / 5 \% \mathrm{CO}_{2}$.

2 Place the slices in a submersion type storage chamber in extracellular solution and let the slices rest for at least 1 hour before an experiment. While the slices are resting, prepare the electrophysiology/imaging setup for the recording session (see Equipment Setup). Ensure that the well-bubbled extracellular solution has been circulating for a minimum of 20 minutes prior to recording at $\sim 3 \mathrm{ml} / \mathrm{min}$.

\section{Cell patching $\bullet$ Timing: 10-30 minutes (depending on slice quality)}

3 Thaw a $200 \mu \mathrm{L}$ aliquot of the intracellular solution (see Intracellular Solution) and supplement this with OGB-1 and Alexa $59410 \mathrm{mM}$ stock solutions to final concentrations of $200 \mu \mathrm{M}$ and $50 \mu \mathrm{M}$ respectively. Filter the internal solution using a filter tube (Corning Spin-X, $0.22 \mu \mathrm{m}$, Mfr. no. 8161) and store the filtered solution on ice during the recording session.

$4 \quad$ Load a 2.5-3.5 M $\Omega$ patch pipette (see Electrophysiology Equipment) with $5 \mu \mathrm{L}$ of the indicator-containing internal solution.

5 Place the brain slice from Step 2 on the microscope and localise a target cell of interest using infrared DIC imaging for slice preparations; conventional DIC imaging could be used for experiments in (translucent) cell cultures. 
6 Patch the neurone or astrocyte of interest with 2.5-3.5 $\mathrm{M} \Omega$ patch pipettes filled with the indicator-containing internal solution in the whole-cell patch-clamp configuration. The present protocol requires hands-on experience with patch-clamp techniques (which vary among different laboratories) and usually involves training under personal supervision. Detailed information about various patch-clamp electrophysiology methods and procedures suitable for the present protocol can be found in the literature ${ }^{53-55}$, including that with special relevance to calcium imaging ${ }^{56}$.

\section{Experimental recording $\bullet$ Timing 60-120 minutes depending on tasks}

$7 \quad$ Following break-in to the target cell, wait for 30 minutes for indicator dyes to equilibrate throughout the dendritic tree, if recording from distal axonal processes a longer duration (40-60 $\mathrm{min}$ ) is required.

CRITICAL STEP Throughout recordings, ensure adequately detailed notes are taken, either electronically or in a lab book. Electrophysiology, imaging and lifetime imaging data will be acquired on separate file systems and the ability to readily correlate these data requires that one has the information to readily locate the relevant files from all systems.

CRITICAL STEP As with all imaging experiments the protocol used depends on the nature of the experiment and the trade-off between preserving the spatial and temporal resolution of the data must be taken into account. FLIM imaging adds a further constraint to the latter as photon counts at each time point must be sufficient for the NTC or gated intensity methods to be applied (Fig. 4a). For data shown in Fig. 4 and Fig. 5 the following protocol was carried out.

8 Monitor and record $\left[\mathrm{Ca}^{2+}\right]$ to achieve your experimental objectives. To map intracellular landscapes of nanomolar resting $\left[\mathrm{Ca}^{2+}\right]$ follow Option $\mathrm{A}$, to record rapid transient $\left[\mathrm{Ca}^{2+}\right]$ changes, follow Option B. 
(A) Recording and mapping nanomolar resting $\left[\mathrm{Ca}^{2+}\right]$ [Troubleshooting]

(i) Set the TCSPC system to the FIFO mode for time lapsed 2D (tXYT) imaging or simple summation mode to get a time-lapse FLIM dataset.

(ii) On the 2PLSM set appropriate zoom and scanning positions to visualise the target cell, for 3D imaging (time-lapse images involving a z-stack of frames for each acquisition time point) also set upper and lower z positions. Set electrophysiology recording in Gap-free mode and initiate the scanning sequence.

(iii) Monitor pixel photon count in the dimmest part of the $\mathrm{ROI}$ and correct for drift during acquisition.

(iv) Stop acquisition once photon count has reached near 1000/pixel in the dimmest part of the ROI.

CRITICAL STEP: Ensure a resting period of 2-3 seconds is afforded between scans to avoid phototoxicity during long acquisitions. For 2D acquisitions this is especially important as the same focal plane will be continually scanned.

(B) Recording transient $\left[\mathrm{Ca}^{2+}\right]$ changes [Troubleshooting]

(i) On the two-photon laser scanning microscope, set the appropriate zoom and scanning positions to visualise the target structure.

(ii) Determine the optimal line scan mode (straight or spiral) to maximise the number of photons counted and achieve good temporal resolution.

(iii) Set the TCSPC system to FIFO mode to collect single photon counting data in cycles started by an external trigger.

(iv) Set electrophysiology recording/stimulation protocol to start on external trigger 
(v) Record multiple trials of line scans to have sufficient photon count in the averaged data.

CRITICAL STEP: Ensure a resting period of 30-40 seconds is afforded between line scans to avoid phototoxicity and to ensure no short-term plasticity processes are triggered which may alter the response between trials.

\section{Arranging data storage $\bullet$ Timing 10-20 minutes}

9 Save TCSPC data in appropriate formats (spc for FIFO, sdt for 2D data from Becker \& Hickl and ptu from PicoQuant) if not already automatically saved by the TCSPC software. Ensure the file or directory names can be readily matched between related TCSPC, imaging and electrophysiology data files. Backup the files and transfer to a separate PC for analysis. [Troubleshooting]

\section{FLIM data analyses}

10 Analyse the obtained data using a normal photon count (NTC) analysis (Option A), gated intensity analysis for signal time course (Option B), or Gated intensity analysis for autofluorescence removal (Option C). See Experimental Design for more details on the different analysis types.

\section{(A) Normalised photon count (NTC) analysis • Timing 20-30 min depending on the task}

(i) Load the acquired data into the analysis programme (FIMAS in our case). In case .sdt files have been generated, image analysis software such as ImageJ or Fiji can also be used. [Troubleshooting]

(ii) If the data set is the time course of the fluorescence decay averaged over one $\mathrm{ROI}$ of interest skip to step (vi) below. If the data set consists of a time-lapse 
images, first select a ROI (e.g., a specific region inside the cell) for FLIM analyses.

(iii) Inspect the fluorescence decay trace at various spatial and temporal binning levels at the dimmest part of the ROI selected for FLIM analyses.

(iv) Choose the binning level which gives sufficient photon counts (at least 5-10 photons towards the end of the integration interval) to avoid estimation uncertainties and reduce errors (Fig. 4a). [Troubleshooting]

(v) Apply the same binning level $(\mathrm{N})$ on a pixel by pixel basis to the entire $\mathrm{ROI}$ of interest (e.g., the entire cell structure, excluding the surrounding background), with each resulting pixel containing sum of data of $\mathrm{N} \times \mathrm{N}$ neighbouring pixels.

(vi) Normalise resulting fluorescence decay traces on a pixel by pixel basis for all resulting pixels, by dividing the decay trace by its maximum.

(vii) Integrate each trace from its peak position to a chosen fixed interval postpeak, to obtain the NTC value. In the present example, a 9 ns interval was chosen because it provided a maximal increase of the signal-to-noise ratio in NTC counts.

(viii) Transform the resulting NTC map/value to estimate free calcium map/value using the inverted logistic function, as calculated during the calibration process.

(ix) Apply false colour mapping, for illustration purposes.

(x) Scale each pixel intensity in the estimated free calcium colour map using the origin photon count number so that cellular structures are easier to visualise.

(xi) Inspect the colour map for the ROI (e.g. soma, healthy spines and boutons).

(xii) Select a ROI to produce a single FLIM decay trace for a specific ROI by summing all raw FLIM data within that ROI. 
(xiii) Repeat steps (xi-xii) for each ROI to obtain the (time-lapse) map of estimated free $\left[\mathrm{Ca}^{2+}\right]$.

(B) Gated intensity analysis for signal time course • Timing 20-30 min depending on the task

(i) Load the acquired data into the analysis programme (FIMAS in our case). In case .sdt files have been generated, image analysis software such as ImageJ or Fiji can also be used. [Troubleshooting].

(ii) Bin in the $X$ dimension of a line scan from entire $\mathrm{ROI}$ selected from fast frame scans (Fig. 5b) to produce a 2D dataset (matrix) with the fluorescence decay photon count data (decay time course) stored for each acquisition time point in time-lapsed imaging.

(iii) Bin in dimension T (global time in time-lapse imaging) in order to obtain fluorescent decay traces in accordance with the acceptable noise level (Fig. 4a, see Assessing FLIM readout variability and measurement error below).

(iv) Find the peak of each decay trace, as illustrated in Fig. 5c (Peak).

(v) Normalise all decay traces to their respective peak values as per step (iv).

(vi) Integrate from the Peak time point + time $T$ till the end of the decay traces (Fig. $5 \mathrm{c}-\mathrm{d}$ ), where $\tau$ is average fluorescent lifetime (for instance, $\tau \sim 3.8 \mathrm{~ns}$ for OGB1 in low basal $\mathrm{Ca}^{2+}$ ). [Troubleshooting]

(C) Gated intensity analysis for autofluorescence removal • Timing 20-30 min depending on the task 
(i) Load the acquired 2D image data into the analysis programme (FIMAS in our case). In case .sdt files have been generated, image analysis software such as ImageJ or Fiji can also be used. [Troubleshooting].

(ii) In the image FLIM data file, check if the decay time constant $\tau$ within the ROI displaying greatest autofluorescence (example shown by arrow and circle in Fig. 6a) is significantly shorter than that of the functional fluorophore .

(iii) Vary the gating interval (e.g., starting with 'half-gated', as shown Fig. 5c) while monitoring the corresponding image, until the image autofluorescence signal appears at its dimmest (Fig. 6b).

(iv) Find the peak of each fluorescence decay trace, as illustrated in Fig. 5c (Peak).

(v) Normalise all decay traces to their respective peak values determined as per step (iv).

(vi) Apply small spatial binning if necessary to reduce noise post processing.

(vii) Integrate decay traces on a pixel by pixel basis during the chosen (optimal) time-gated interval as per step (iii), to produce a resulting intensity image map, as shown in Fig. 6 a.

(viii) Scale the resulting map by its original intensity map (Fig. 6a) to produce the final image (Fig. 6b), to match the brightness of the original and time-gated images, for comparison purposes.

\section{TROUBLESHOOTING}

Troubleshooting advice can be found in Table 4. 


\section{TIMING}

Set up FLIM system onto existing fluorescent scanning microscope: $2-5 d$

Calibration procedure (solution preparation, measurement, analysis, as detailed Supplementary Fig. 1): 1-2 d

Step 1, Acute slice preparation and storage: 10-20 min

Step 2, Slice resting and transfer to the recording chamber: 80-100 min

Step 3, Preparing fresh intracellular solution: 10-20 min

Step 4, Pipette loading: 1-2 min

Step 5, Locating the cell of interest under the microscope: 5-10 min

Step 6, Approaching and patching the cell in whole-cell mode: 5-30 min

Step 7, Pipette dye equilibration: 30-40 min

Step 8 (A), Recording resting $\left[\mathrm{Ca}^{2+}\right]: 30 \mathrm{~min}-3 \mathrm{hr}$

Step 8 (B), Recording transient $\left[\mathrm{Ca}^{2+}\right]$ changes: $30 \mathrm{~min}-3 \mathrm{hr}$

Step 9, Arranging data storage: 10-20 min

Step 10 (A), Analysing NTC data: 20-30 min

Step $10(B)$, Analysing gated intensity $\left[\mathrm{Ca}^{2+}\right]$ time course data: 5-20 min

Step $10(C)$, Removing autofluorescence signal: 5-10 min

\section{ANTICIPATED RESULTS}

Normalised Total Count The output of OGB-1 calibration against $\left[\mathrm{Ca}^{2+}\right]$ should produce almost noiseless fluorescent decay traces (Fig. 2a) as the photon collection time is not limited by the experimental conditions. Applying the normalised total count (NTC) method to these traces and plotting the outcome against the estimated 
$\left[\mathrm{Ca}^{2+}\right]$ produces a near-perfect fit for logistic function (Fig. 2b). This remains unchanged in the presence of other environmental factors (temperature, $\mathrm{pH}$, viscosity, the presence of $\mathrm{Mg}^{2+}, \mathrm{Zn}^{2+}$, or the ubiquitous protein actin) varied within the physiological range pertinent to hippocampal pyramidal neurons and astroglia ${ }^{27}$. Similar results with different fitted values of logistic function are expected for the entire OGB family of fluorescent indicators.

\section{Assessing FLIM readout variability and measurement error There are several} inter-related sources of error or noise in $\left[\mathrm{Ca}^{2+}\right]$ FLIM measurements when using the NTC method. Firstly, the NTC estimation variability during acquisition directly depends on the acquisition time (or the total number of detected photons). Increasing the number of detected photons increases the signal-to-noise $(\mathrm{S} / \mathrm{N})$ ratio of the recorded fluorescence (decay peak signal over the background signal) thus decreasing the measurement error of NTC readout. An example recorded in calibration settings $\left(\left[\mathrm{Ca}^{2+}\right]\right.$ clamped at $75 \mathrm{nM}$ ) indicates that with $\mathrm{S} / \mathrm{N}$ ratios of 100 , 1000 and 4000 one obtains the NTC estimate with a coefficient of variation (CV; standard deviation of the mean) of $11 \%, 6 \%$ and $3 \%$, respectively (Fig. 2c, d). With $\left[\mathrm{Ca}^{2+}\right]=75 \mathrm{nM}$, these $\mathrm{CV}$ values correspond to the absolute measurement errors of $8.25 \mathrm{nM}, 4.5 \mathrm{nM}$, and $2.25 \mathrm{nM}$, respectively. The user has to empirically determine imaging settings (laser power, dye concentration, $\mathrm{ROI}$ size, etc.) and acquisition time that would minimise the measurement error while meeting the requirements of the imaging task. In the type of imaging experiments presented here, it has been feasible to consistently achieve the relative OGB-1 NTC measurement error of $5 \%$ or less. Therefore, in these settings the difference in $\left[\mathrm{Ca}^{2+}\right]$ levels (among different ROls detected with the NTC approach, within or among cells) of $5 \%$ or below could be considered statistically insignificant. Conversely, greater differences in NTC readout are likely to reflect genuine differences in local $\left[\mathrm{Ca}^{2+}\right]$.

To test whether the measurement accuracy, as described above, depends on the fluorescent indicator and its spectral properties (and hence is OGB-1 specific), we 
measured NTC variability for two $\mathrm{Ca}^{2+}$-independent fluorophores emitting at different wavelengths: Alexa Fluor 647 (puffed from a micropipette), and Alexa Flour 594 (loaded into a live CA3 pyramidal cell) (Fig. 3a). In both cases, a CV of $~ 5 \%$ was achieved in imaging settings similar to those in OGB-1 experiments (Fig. $3 \mathrm{~b}$ ), thus reflecting the expected measurement error in NTC readout ${ }^{27}$. The uniformity of the instrumental error across different indicators in our experiments suggests that the present method could be reliably used with indicators other than OGB-1, as long as they display the fluorescence decay sensitive to $\left[\mathrm{Ca}^{2+}\right]$.

Our experimental observations in situ documented five-fold variations in the intracellular basal $\left[\mathrm{Ca}^{2+}\right]$ among and within cells, roughly between $\sim 30 \mathrm{nM}$ and $\sim 150$ $\mathrm{nM}^{27,28}$. The $5 \%$ NTC variability, or relative measurement error, estimated here thus corresponds to the absolute error (SD) in concentration measurements ranging between $\pm 1.5 \mathrm{nM}$ and $\pm 7.5 \mathrm{nM}$ (the value scaling with the $\left[\mathrm{Ca}^{2+}\right]$ value). This range of error indicates a biological (non-artefactual) source of variations in $\left[\mathrm{Ca}^{2+}\right]$ detected here. The fact that the within-cell $\left[\mathrm{Ca}^{2+}\right]$ variability detected with NTC readout substantially exceeds the measurement error of the NTC readout can be illustrated by a direct comparison of the NTC distributions obtained for the $\mathrm{Ca}^{2+}$-independent AF594 and the $\mathrm{Ca}^{2+}$-dependent OGB-1 in the same live cell. When both indicators were imaged simultaneously in the same principal neuron in situ, the OGB-1 NTC distribution was much wider than the AF594 one (Fig. 3c-d) even though the measurement errors for either indicator were similar ( 5\%) (compare Fig. $2 d$ and Fig. 3b).

A further illustration of the variability (noise) assessment in monitoring steady-state resting $\left[\mathrm{Ca}^{2+}\right]$ inside a hippocampal CA3 pyramidal cell depicts the typical OGB-1 fluorescence decay that corresponds to what we considered noise acceptability criterion, i.e. when the photon count values in the decay time course (Fig. 4a, black dots) deviated by $<5 \%$ from the best-fit exponential decay (Fig. $4 a$, grey curve). When the photon counts deviated by $>5 \%$ but with a clearly convergent decay fit (Fig. 4a, red dots, pink line), caution should be taken when interpreting the results. 
When data cannot generate a convergent fit (Fig. 4a, blue dots), the imaging has to be repeated at more favourable settings.

Our empirical observations suggest that the pixel-by-pixel analysis and mapping produce reliable and reproducible data when the noise criteria are met across $>99 \%$ of the $\mathrm{ROI}$ (Fig. $4 b$ ). If $>1 \%$ pixels within the cell $\mathrm{ROI}$ fail the noise criteria, the data mapping should be discarded and an alternative $\mathrm{ROI}$ analysis pursued where one could increase photon count for ROI by accumulating larger amount of pixels.

We note that the above considerations and the corresponding examples (Figs. 2-4) illustrate how to evaluate and possibly control the FLIM-based $\left[\mathrm{Ca}^{2+}\right]$ measurement error (or precision) in the imaging experiments performed in live cells. The actual degree of $\left[\mathrm{Ca}^{2+}\right]$ readout variability will depend on a particular instrumental arrangement and the imaging settings in the host laboratory.

Gated intensity to improve kinetic signal monitoring The gated intensity approach was applied to a hippocampal CA3 pyramidal cell axonal bouton occurring $\sim 100 \mu \mathrm{m}$ away from the soma. Whilst the tracing of an axon from the soma is best performed using the bright Alexa fluorescence (Fig. 5a), the bouton of interest and the adjacent section of the axon could be visualised using OGB-1 only (no additional morphological tracer) at the resting state (Fig. 5b). In this example, we recorded axonal $\left[\mathrm{Ca}^{2+}\right]$ during four bursts of action potentials (10 pulses at $1 \mathrm{~Hz}$ each, soma held in current clamp). The choice of gating intervals, including the tail end (12.5 ns, which is defined by the laser repetition rate of $80 \mathrm{MHz}$ ) and the second half of the decay were tested against the NTC (Fig. 5c-d). The conventional normalised total count (NTC) fluorescence intensity monitoring displayed noisy fluctuations and a photobleaching trend (Fig. 5d). Once the gated intensity has been applied, the signal-to-noise ratio of the transient improves substantially, with the half-gated protocol providing the optimal choice (Fig. 5 d). Combined with $\left[\mathrm{Ca}^{2+}\right]$ calibration data (recalculated for the half-gated photon count using existing raw data, Supplementary Fig. 2), this approach produced the time course of absolute $\left[\mathrm{Ca}^{2+}\right]\left(\mathrm{Ca}^{2+}\right.$ buffer- 
equilibrated, see ${ }^{27}$ ) (Fig. 5e). Overall, in conditions of a typical fluorescence imaging experiment, the half-gated intensity method improves the $\Delta \mathrm{F} / \mathrm{F}_{0}$ dramatically (Fig. 5f), even though it does not provide the linearity and direct readout of $\left[\mathrm{Ca}^{2+}\right]$ of the NTC approach. Note that the same gating interval has to be used for valid comparisons among separate recording sessions.

Gated intensity to remove autofluorescence The gated intensity method can also be used to remove autofluorescence, which normally has a relatively shorter lifetime but emits over the entire visible spectrum $(400-700 \mathrm{~nm})$. This approach can be combined with having a $\mathrm{Ca}^{2+}$ indicator, such as OGB-1, and simultaneously achieve autofluorescence background reduction and $\Delta \mathrm{F} / \mathrm{F}_{0}$ improvement.

Tissue autofluorescence could contaminate $\mathrm{Ca}^{2+}$ dependent OGB-1 fluorescence readout in live cell experiments in situ, but it can be substantially reduced by removing the short-lifetime signal component in a half-gated-intensity approach (Fig. 6a). A similar method can be applied to remove artefactual autofluorescence emission from the cell image in the morphological tracer (AF594) channel (Fig. 6b).

\section{AUTHOR CONTRIBUTION STATEMENTS}

K.Z. designed and set up the optical system arrangements, software, and analysed the data; K.Z. and D.A.R. conceived and designed the experiments; T.J. and K.Z. performed the experiments; K.Z., T.J. and D.A.R. wrote the manuscript.

\section{ACKNOWLEDGEMENTS}

This work was supported by a Wellcome Trust Principal Fellowship (101896), European Research Council Advanced Grant (323113 NETSIGNAL), Russian Science Foundation grant (15-14-30000, computing cluster setup), FP7 ITN (606950 EXTRABRAIN), European Research Council Proof-of-Concept Grant (to D.A.R.). 


\section{COMPETING FINANCIAL INTERESTS}

The authors declare no competing financial interests.

INCLUDED: SUPPLEMENTARY FIGURES 1-2 


\section{REFERENCES}

1 Katz, B. \& Miledi, R. The role of calcium in neuromuscular facilitation. J. Physiol. 195, 481-492 (1968).

2 Lisman, J. A mechanism for the Hebb and the anti-Hebb processes underlying learning and memory. Proc. Natl. Acad. Sci. USA 86, 9574-9578 (1989).

3 Nishiyama, M., Hong, K., Mikoshiba, K., Poo, M. M. \& Kato, K. Calcium stores regulate the polarity and input specificity of synaptic modification. Nature 408 , 584-588 (2000).

4 Perea, G., Navarrete, M. \& Araque, A. Tripartite synapses: astrocytes process and control synaptic information. Trends Neurosci 32, 421-431 (2009).

5 Halassa, M. M. \& Haydon, P. G. Integrated brain circuits: astrocytic networks modulate neuronal activity and behavior. Annu Rev Physiol 72, 335-355 (2010).

6 Di Castro, M. A. et al. Local Ca2+ detection and modulation of synaptic release by astrocytes. Nature Neurosci. 14, 1276-1284 (2011).

7 Shigetomi, E. et al. Imaging calcium microdomains within entire astrocyte territories and endfeet with GCaMPs expressed using adeno-associated viruses. J Gen Physiol 141, 633-647 (2013).

8 Yasuda, R., Sabatini, B. L. \& Svoboda, K. Plasticity of calcium channels in dendritic spines. Nat Neurosci 6, 948-955 (2003).

9 Wilms, C. D. \& Hausser, M. Lighting up neural networks using a new generation of genetically encoded calcium sensors. Nat Methods 6, 871-872 (2009).

10 Chen, T. W. et al. Ultrasensitive fluorescent proteins for imaging neuronal activity. Nature 499, 295-300 (2013).

11 Grynkiewicz, G., Poenie, M. \& Tsien, R. Y. A new generation of Ca2+ indicators with greatly improved fluorescence properties. J Biol Chem 260, 3440-3450 (1985).

12 Tsien, R. Y. Fluorescent probes for cell signaling. Annu Rev Neurosci 12, 227253 (1989).

13 Maravall, M., Mainen, Z. F., Sabatini, B. L. \& Svoboda, K. Estimating intracellular calcium concentrations and buffering without wavelength ratioing. Biophys J 78, 2655-2667 (2000).

14 Grienberger, C. \& Konnerth, A. Imaging Calcium in Neurons. Neuron 73, 862885 (2012). 
15 Shigetomi, E., Kracun, S., Sofroniew, M. V. \& Khakh, B. S. A genetically targeted optical sensor to monitor calcium signals in astrocyte processes. Nat Neurosci 13, 759-766 (2010).

16 Scott, R. \& Rusakov, D. A. Main determinants of presynaptic $\mathrm{Ca}^{2+}$ dynamics at individual mossy fiber-CA3 pyramidal cell synapses. J. Neurosci. 26, 7071 7081 (2006).

17 Vyleta, N. P. \& Jonas, P. Loose coupling between $\mathrm{Ca2}+$ channels and release sensors at a plastic hippocampal synapse. Science 343, 665-670 (2014).

18 Faas, G. C., Raghavachari, S., Lisman, J. E. \& Mody, I. Calmodulin as a direct detector of Ca2+ signals. Nature Neurosci. 14, 301-304 (2011).

19 Sylantyev, S., Jensen, T. P., Ross, R. A. \& Rusakov, D. A. Cannabinoid- and lysophosphatidylinositol-sensitive receptor GPR55 boosts neurotransmitter release at central synapses. Proc Natl Acad Sci U S A 110, 5193-5198 (2013).

20 Christie, J. M., Chiu, D. N. \& Jahr, C. E. Ca(2+)-dependent enhancement of release by subthreshold somatic depolarization. Nature Neurosci. 14, 62-68 (2011).

21 Ueda, Y., Kwok, S. \& Hayashi, Y. Application of FRET probes in the analysis of neuronal plasticity. Frontiers in Neural Circuits 7 (2013).

22 Koldenkova, V. P. \& Nagai, T. Genetically encoded Ca2+ indicators: Properties and evaluation. Biochimica Et Biophysica Acta-Molecular Cell Research 1833, 1787-1797 (2013).

23 Mikuni, T., Nishiyama, J., Sun, Y., Kamasawa, N. \& Yasuda, R. HighThroughput, High-Resolution Mapping of Protein Localization in Mammalian Brain by In Vivo Genome Editing. Cell 165, 1803-1817 (2016).

24 Wilms, C. D. \& Eilers, J. Photo-physical properties of Ca2+-indicator dyes suitable for two-photon fluorescence-lifetime recordings. J Microsc 225, 209213 (2007).

25 Wilms, C. D., Schmidt, H. \& Eilers, J. Quantitative two-photon Ca2+ imaging via fluorescence lifetime analysis. Cell Calcium 40, 73-79 (2006).

26 Kuchibhotla, K. V., Lattarulo, C. R., Hyman, B. T. \& Bacskai, B. J. Synchronous hyperactivity and intercellular calcium waves in astrocytes in Alzheimer mice. Science 323, 1211-1215 (2009).

27 Zheng, K. et al. Time-resolved imaging reveals heterogeneous landscapes of nanomolar $\mathrm{Ca}^{2+}$ in neurons and astroglia. Neuron 88, 277-288 (2015).

28 Jennings, A. et al. Dopamine elevates and lowers astroglial $\mathrm{Ca} 2+$ through distinct pathways depending on local synaptic circuitry. Glia 65, 447-459 (2017). 
29 Jensen, T. P., Zheng, K., Tyurikova, O., Reynolds, J. P. \& Rusakov, D. A. Monitoring single-synapse glutamate release and presynaptic calcium concentration in organised brain tissue. Cell Calcium 64, 102-108 (2017).

30 Lakowicz, J. R. Principles of Fluorescence Spectroscopy (3rd Edition). (Springer, 2006).

31 Denk, W., Strickler, J. H. \& Webb, W. W. Two-photon laser scanning fluorescence microscopy. Science 248, 73-76 (1990).

32 Denk, W. et al. Anatomical and functional imaging of neurons using 2-photon laser scanning microscopy. J Neurosci Methods 54, 151-162 (1994).

33 Rich, R. M. et al. Elimination of autofluorescence background from fluorescence tissue images by use of time-gated detection and the AzaDiOxaTriAngulenium (ADOTA) fluorophore. Anal Bioanal Chem 405, 2065-2075 (2013).

34 Striker, G., Subramaniam, V., Seidel, C. A. M. \& Volkmer, A. Photochromicity and fluorescence lifetimes of green fluorescent protein. J Phys Chem B 103, 8612-8617 (1999).

35 Gee, K. R. et al. Chemical and physiological characterization of fluo-4 Ca2+indicator dyes. Cell Calcium 27, 97-106 (2000).

36 Schnell, S. A., Staines, W. A. \& Wessendorf, M. W. Reduction of lipofuscinlike autofluorescence in fluorescently labeled tissue. J Histochem Cytochem 47, 719-730 (1999).

37 Baschong, W., Suetterlin, R. \& Laeng, R. H. Control of autofluorescence of archival formaldehyde-fixed, paraffin-embedded tissue in confocal laser scanning microscopy (CLSM). Journal of Histochemistry \& Cytochemistry 49, 1565-1571 (2001).

38 Neumann, M. \& Gabel, D. Simple method for reduction of autofluorescence in fluorescence microscopy. J Histochem Cytochem 50, 437-439 (2002).

39 Regehr, W. G. \& Tank, D. W. Calcium concentration dynamics produced by synaptic activation of CA1 hippocampal pyramidal cells. J Neurosci 12, $4202-$ 4223 (1992).

40 Bers, D. M. Calcium cycling and signaling in cardiac myocytes. Annu Rev Physiol 70, 23-49 (2008).

41 Agronskaia, A. V., Tertoolen, L. \& Gerritsen, H. C. Fast fluorescence lifetime imaging of calcium in living cells. J Biomed Opt 9, 1230-1237 (2004).

42 Zheng, K. Y. et al. Nanoscale diffusion in the synaptic cleft and beyond measured with time-resolved fluorescence anisotropy imaging. Scientific Reports 7, 42022 (2017). 
43 Despa, S., Steels, P. \& Ameloot, M. Fluorescence lifetime microscopy of the sodium indicator sodium-binding benzofuran isophthalate in HeLa cells. Anal Biochem 280, 227-241 (2000).

44 Becker, W. The bh TCSPC Handbook. 6 edn, (Becker and Hickl GmbH, 2015).

45 Liu, M. W. et al. Instrument Response Standard in Time-Resolved Fluorescence Spectroscopy at Visible Wavelength: Quenched Fluorescein Sodium. Appl Spectrosc 68, 577-583 (2014).

46 Minta, A., Kao, J. P. \& Tsien, R. Y. Fluorescent indicators for cytosolic calcium based on rhodamine and fluorescein chromophores. J Biol Chem 264, 81718178 (1989).

$47 \quad$ Paredes, R. M., Etzler, J. C., Watts, L. T., Zheng, W. \& Lechleiter, J. D. Chemical calcium indicators. Methods 46, 143-151 (2008).

48 Lalo, U., Palygin, O., North, R. A., Verkhratsky, A. \& Pankratov, Y. Agedependent remodelling of ionotropic signalling in cortical astroglia. Aging Cell 10, 392-402 (2011).

49 Scott, R., Ruiz, A., Henneberger, C., Kullmann, D. M. \& Rusakov, D. A. Analog modulation of mossy fiber transmission is uncoupled from changes in presynaptic Ca2+. J. Neurosci. 28, 7765-7773 (2008).

50 Andersen, P., Bliss, T. V. \& Skrede, K. K. Lamellar organization of hippocampal pathways. Exp. Brain Res. 13, 222-238 (1971).

51 Schwartzkroin, P. A. Characteristics of CA1 neurons recorded intracellularly in the hippocampal in vitro slice preparation. Brain Res. 85, 423-436 (1975).

52 Teyler, T. J. Brain slice preparation: hippocampus. Brain research bulletin 5, 391-403 (1980).

53 Bischofberger, J., Engel, D., Li, L., Geiger, J. R. P. \& Jonas, P. Patch-clamp recording from mossy fiber terminals in hippocampal slices. Nature Protocols 1, 2075-2081 (2006).

54 Davie, J. T. et al. Dendritic patch-clamp recording. Nature Protocols 1, 12351247 (2006).

55 Molleman, A. Patch clamping: an introductory guide to patch clamp electrophysiology. John Wiley \& Sons (2003).

56 Yasuda, R. et al. Imaging calcium concentration dynamics in small neuronal compartments. Sci STKE 2004, pl5 (2004).

57 Williams, P. A., Larimer, P., Gao, Y. \& Strowbridge, B. W. Semilunar granule cells: glutamatergic neurons in the rat dentate gyrus with axon collaterals in the inner molecular layer. J Neurosci 27, 13756-13761 (2007). 
58 Schmidt-Hieber, C., Jonas, P. \& Bischofberger, J. Subthreshold dendritic signal processing and coincidence detection in dentate gyrus granule cells. $J$ Neurosci 27, 8430-8441 (2007).

59 Scimemi, A. et al. Tonic GABA(A) receptor-mediated currents in human brain. Eur J Neurosci 24, 1157-1160 (2006). 


\section{FIGURE LEGEND}

\section{Figure 1. Schematic of the time-resolved imaging system.}

A diagram depicting key components of the imaging system, as described in the Introduction. Notations: BS1, beam splitter; P1, periscope unit (fast laser mirrors with low group velocity dispersion); D1, excitation dichroic; D2, emission dichroic; F1 and F2, emission filters; DET1 and DET2, detectors. See Equipment section for further detail.

\section{Figure 2. Photon count versus free calcium calibration and experimental noise} assessment.

a, Calibration example: Normalised fluorescent decay traces for calcium calibration solutions; shadow indicates integration (area-under-the-curve) interval, to be related to the peak value (arrow). The data show significant decay in sensitivity to $\mathrm{Ca}^{2+}$ concentration within the range of $0-300 \mathrm{nM}$; data are acquired at $33^{\circ} \mathrm{C}$

b, Normalised total count values (circles) as described by the integration of normalised fluorescent decays (shown in a); when plotted against log $\left[\mathrm{Ca}^{2+}\right]$ it can be accurately fitted with a logistic function (solid line) with respect to $\left[\mathrm{Ca}^{2+}\right]$ values, as detailed in steps 41-42 of the Calibration Procedure (Supplementary Fig. 1).

c, Experimental noise assessment: examples of image recordings of OGB-1 calibration solution ([ $\left.\mathrm{Ca}^{2+}\right]$ clamped at $75 \mathrm{nM} \mathrm{Ca}^{2+}$; intensity grey scale), for three different photon collection times (otherwise imaged at the same settings) that correspond to the three signal-to-noise ratios $(\mathrm{S} / \mathrm{N})$, as indicated. The $\mathrm{S} / \mathrm{N}$ ratios were calculated as the peak intensity of fluorescence decay traces over background intensity (no excitation) under the same conditions. 
d, The scatter (noise) of documented NTC values over the ROI pixels for the three scenarios depicted in c; the mean NTC value is normalised to 1.0 for illustration purposes. The three NTC scatters correspond to a coefficient of variation (CV, standard deviation over mean) of $11 \%, 6 \%$, and $3 \%$ (S/N: 100,1000 , and 4000 , respectively).

Figure 3. FLIM readout variability assessment in vitro and in situ for $\mathrm{Ca}^{2+}$ independent indicators and OGB-1.

a, A FLIM readout map for Alexa Fluor 647 (AF647) puffed into water from a patch pipette (left), and a fragment of a CA3 pyramidal cell (primary dendrite) filled with AF594 and FLIM-mapped (right). False colour scale (NTC, arbitrary values) applies as indicated; brightness encodes signal intensity. Note: AF647 has smaller NTC values compared to AF594 because the former has a much shorter lifetime.

b, NTC value scatters over the entire image regions occupied by the fluorescent indicator (non-zero-value pixels), for the two recordings shown in a, as indicated; mean NTC value in both cases is normalised to one for comparison purposes (original mean values were, in relative units, 0.114 and 0.379, for AF647 and AF594, respectively). The scatters correspond to CV (error) of $\sim 5 \%$ for imaging with $\mathrm{Ca}^{2+}$-independent indicators (AF647 and AF 594).

c, An example of FLIM readout maps depicting a CA3 pyramidal cell in the OGB-1 (left) and AF594 (right) emission channels. False colour scale (NTC, arbitrary values) applies as indicated; brightness encodes signal intensity.

d, NTC value scatters over the image regions occupied by the cellular fluorescence (non-zero-value pixels; pipette excluded), for the two channels shown in c, as indicated; mean NTC value in both cases is normalised to one for comparison purposes (original mean values were, in relative units, 0.11 and 0.36 , 
for OGB-1 and AF594, respectively). The OGB-1 scatter (CV 20\%) is much wider compared to that of AF594 (CV 8\%, the value), thus reflecting a heterogeneous distribution of basal $\left[\mathrm{Ca}^{2+}\right]$ across the cell. Note that the AF594 NTC distribution is skewed to the left (and its CV is higher than that shown in Fig. 3b) due to the presence of the contaminating autofluorescence in the image (see Fig. 6): in contrast, a cell dendritic fragment shown in a (right panel) at higher magnification contains no detectable autofluorescence areas, hence its NTC scatter for AF594 has no visible skewness.

\section{Figure 4. Illustration of a normalised total count (NTC) technique to map intracellular $\left[\mathrm{Ca}^{2+}\right]$ in the plane of view.}

a, Example of normalised fluorescence decay traces, with individual photon count values (dots) and the three-exponent fitting curves (solid lines, fitting used for data noise estimation only), shown at different noise levels. The fully acceptable level is shown by black dots ( $\mathrm{S} / \mathrm{N}$ ratio $~ 800$ ): individual values deviate from the fitting curve (grey) by $<5 \%$. The noisy data (red dots, pink curve) deviate by $>5 \%$ from the bestfit decay, and the rejected data (blue dots) cannot generate converged fitting.

b, Example of a basal $\left[\mathrm{Ca}^{2+}\right]$ map in a hippocampal CA3 pyramidal cell obtained using the NTC analysis technique. The map reveals subtle variations in $\left[\mathrm{Ca}^{2+}\right]$ levels across cellular compartments. False colour scale for $\left[\mathrm{Ca}^{2+}\right]$ range; brightness, signal intensity; scale bar, $10 \mu \mathrm{m}$. Note that the higher residual $\left[\mathrm{Ca}^{2+}\right]$ in the pipette tip is likely because it has been moved through a bath medium containing $2 \mathrm{mM}$ $\left[\mathrm{Ca}^{2+}\right]$ (pipette solution has no $\mathrm{Ca}^{2+}$ buffers).

\section{Figure 5. Improving the $\mathrm{Ca}^{2+}$ signal dynamic range and the signal-to-noise} ratio using the gated intensity approach. 
a, Example of a patched CA3 pyramidal cell, with a traced axon (arrows indicate proximal axonal segments traced from the soma; $\left(\lambda_{x}{ }^{2 P}=800 \mathrm{~nm}\right)$; acute hippocampal slice. Note that in two-photon excitation mode only the small (microscopic) fragments of cell processes can be visualised in the focal plane. Therefore, a full reconstruction of the axon, for illustration purposes, routinely involves 'stitching' together $x y$ projections obtained from multiple local optical $z$ stacks (usually 5-15 $\mu \mathrm{m}$ deep, with 0.5-1.0 $\mu \mathrm{m}$ z-steps) containing imaged axonal fragments, at varied magnification settings, along the axon trajectory ${ }^{16,57,58}$. Rectangular areas of varied average brightness along the axon (near arrows) depict examples of such reconstructed axonal fragments ( $x y$ projections of optical $z$ stacks).

b, An axonal varicosity of a CA3 pyramidal cell (as in a, OGB-1 channel; images of intermediate axonal sections between the soma and the bouton are not shown); the $\mathrm{ROI}$ is outlined with blue dashed line; OGB-1 intensity image.

c, Different fluorescence lifetime gating intervals (integration windows), as indicated, selected to test the effect of gating on the detected axonal $\mathrm{Ca}^{2+}$ signal. Vertical dotted arrow, fluorescence decay constant $r$ at basal $\left[\mathrm{Ca}^{2+}\right]$.

d, Traces show a $\mathrm{Ca}^{2+}$ response in the axon shown in $\mathbf{b}$, using the different gating intervals depicted in c. The cell (held in current clamp) was stimulated to generate four bursts of action potentials (10 pulses at $1 \mathrm{~Hz}$ each), with onsets indicated by arrows. In this example, the 'half-gated' approach produces the highest signal-tonoise ratio.

e, The half-gated approach (seen as optimal in $\mathrm{S} / \mathrm{N}$ recordings depicted in $\mathbf{d}$ ) recalibrated for absolute $\left[\mathrm{Ca}^{2+}\right]$ using the FLIM data sets obtained in the standard calibration routine (as in Fig. $2 a-b$ ). Note that such traces report $\left[\mathrm{Ca}^{2+}\right]$ which is rapidly equilibrated with its local buffers upon $\mathrm{Ca}^{2+}$ entry or efflux. 
f, A comparison summary for the total (standard) intensity (left ordinate; black, $n=5$ ), half-gated intensity (left ordinate; orange, $n=5$ ), and FLIM (right ordinate; red, $n=5$ ) methods of $\left[\mathrm{Ca}^{2+}\right]$ measurement in dendrites of a pyramidal cell (in response to 500 ms burst of spikes at different frequencies, as indicated); dots, data of individual experiments; lines, connecting mean values.

Figure 6. Removing artefactual autofluorescence using the tail gated intensity approach.

a, A live CA3 pyramidal cell (as in Fig. 3c, whole-cell mode, dialysed with OGB-1, pipette fragment is seen) depicted as an OGB-1 intensity image, with the total fluorescence intensity (thus including autofluorescence, + auto). Arrow and dotted circle depict the ROI dominated by the artefactual autofluorescence (unrelated to the patched cell): this $\mathrm{ROI}$ is used to characterise the autofluorescence lifetime.

b, Cell as in a shown as a gated intensity image (lifetime range 5-10 ns, see Fig. 5c). Tissue areas showing prominent autofluorescence are dimmed in the right image whereas the cell $\mathrm{Ca}^{2+}$-dependent OGB-1 fluorescence remains intact; false colour scale; the brightness scale is matched between images for comparison purposes.

${ }^{59}$ Table 1. Basic FLIM properties of common fluorophores.

\begin{tabular}{|l|l|l|l|l|}
\hline Fluorophore & $\begin{array}{l}\lambda_{\text {em }} \text { max, } \\
\mathrm{nm}\end{array}$ & Tfree$_{\text {frens }}$ & Tbound, ns & $\begin{array}{l}\text { Supplier / measurement } \\
\text { Reference }\end{array}$ \\
\hline OGB-1 $\left(\mathrm{Ca}^{2+}\right)$ & 523 & 0.73 & 4 & Thermofisher O6806* \\
\hline OGB-2 $\left(\mathrm{Ca}^{2+}\right)$ & 523 & 0.73 & 4.1 & Thermofisher O6808* \\
\hline Calcium Green 1 $\left(\mathrm{Ca}^{2+}\right)$ & 506 & 0.92 & 3.6 & Thermofisher C3010MP* \\
\hline GFP & 516 & 3.2 & 3.2 & $30 * *$ \\
\hline
\end{tabular}




\begin{tabular}{|c|c|c|c|c|}
\hline Су3 & 568 & 0.3 & 0.3 & Sigma $777315^{\star}$ \\
\hline Cy5 & 666 & 1.0 & 1.0 & Sigma $777323^{*}$ \\
\hline Alexa Fluor 488 & 519 & 4.1 & 4.1 & Thermofisher A10436* \\
\hline Alexa Fluor 594 & 617 & 3.8 & 3.8 & Thermofisher A10438* \\
\hline FITC & 518 & 4.1 & 4.1 & $30 \star *$ \\
\hline Fluorescein & 517 & 4.0 & 4.0 & $30 * *$ \\
\hline Sulphorodamine 101 & 520 & 4.32 & 4.32 & Sigma S7635* \\
\hline Texas Red & 615 & 4.2 & 4.2 & $30 * *$ \\
\hline Sodium Green $\left(\mathrm{Na}^{+}\right)$ & 535 & 1.14 & 2.38 & Thermofisher S6900* \\
\hline $\mathrm{SBFI}\left(\mathrm{Na}^{+}\right)$ & $490-499$ & 0.27 & 0.47 & Thermofisher S1262* \\
\hline $\begin{array}{l}\text { Magnesium Green } \\
\left(\mathrm{Mg}^{2+}\right)\end{array}$ & 506 & 0.98 & 3.63 & Thermofisher M3733* \\
\hline PBFI $\left(K^{+}\right)$ & $507-557$ & 0.47 & 0.72 & Thermofisher P1265MP* \\
\hline
\end{tabular}

Notes: $\lambda_{\text {em }}$ max, wavelength of emission peak; $\tau_{\text {free }}$ and $\tau_{\text {bound, }}$ characteristic fluorescence lifetime for the fluorophore in the absence, and under saturating presence, of the sensitivity agent (relevant to ion sensitive fluorophores, as indicated in brackets in left column); ${ }^{*}$, in house FLIM measurements; ${ }^{* *}$, has not been used /supplied in the present study. 
Table 2. Base $\left[\mathrm{Ca}^{2+}\right]$ calibration solutions compatible with intracellular dialysis

\begin{tabular}{|c|c|c|c|c|}
\hline Constituent & Supplier & M.W. & $\begin{array}{l}\text { Ca LS } \\
(\mathrm{mM})\end{array}$ & $\begin{array}{l}\text { Ca HS } \\
(\mathrm{mM})\end{array}$ \\
\hline $\begin{array}{l}\text { Methanesulphonic } \\
\text { acid }\left(\mathrm{CH}_{3} \mathrm{SO}_{3} \mathrm{H}\right)\end{array}$ & $\begin{array}{l}\text { Sigma M6391 or } \\
471356\end{array}$ & 96.11 & 135 & 135 \\
\hline $\begin{array}{l}\text { HEPES, titrate } \\
\left(\mathrm{C}_{8} \mathrm{H}_{18} \mathrm{~N}_{2} \mathrm{O}_{4} \mathrm{~S}\right)\end{array}$ & Sigma H3375-500G & 238.3 & 10 & 10 \\
\hline $\begin{array}{l}\text { Tris-Phosphocreatine } \\
\left(\mathrm{C}_{4} \mathrm{H}_{8} \mathrm{~N}_{3} \mathrm{Na}_{2} \mathrm{O}_{5} \mathrm{P}\right)\end{array}$ & Sigma P7936-5G & 453.4 & 10 & 10 \\
\hline $\begin{array}{l}\text { di-Na ATP } \\
\left(\mathrm{C}_{10} \mathrm{H}_{14} \mathrm{~N}_{5} \mathrm{Na}_{2} \mathrm{O}_{1} 3 \mathrm{P}_{3}\right)\end{array}$ & Sigma A2383-5G & 551.1 & 4 & 4 \\
\hline $\begin{array}{l}\text { di-Na GTP } \\
\left(\mathrm{C}_{10} \mathrm{H}_{16} \mathrm{~N}_{5} \mathrm{O}_{14} \mathrm{P}_{3}\right) \\
(11 \% \mathrm{Na})\end{array}$ & Sigma G8877-250MG & 523.18 & 0.4 & 0.4 \\
\hline $\mathrm{MgCl}_{2}$ & Sigma M2670-G500 & 203.3 & 4 & $4^{*}$ \\
\hline $\begin{array}{l}\text { BAPTA } \\
\left(\mathrm{C}_{22} \mathrm{H}_{24} \mathrm{~N}_{2} \mathrm{O}_{10}\right)\end{array}$ & Sigma A4926 & 476.43 & 10 & 5 \\
\hline $\begin{array}{l}\text { BAPTA } \\
\text { tetrapotassium salt } \\
\left(\mathrm{C}_{22} \mathrm{H}_{20} \mathrm{~K}_{4} \mathrm{~N}_{2} \mathrm{O}_{10}\right)\end{array}$ & Invitrogen B1204 & 628.8 & 0 & 5 \\
\hline $\mathrm{CaCl}_{2}\left(\mathrm{CaCl}_{2} \times 2 \mathrm{H}_{2} \mathrm{O}\right)$ & Sigma C3881-1KG & 147.02 & 0 & $10^{*}$ \\
\hline $\mathrm{KCl}$ & Sigma P9333-500G & 74.55 & 20 & 0 \\
\hline $\begin{array}{l}\text { Oregon Green } 488 \\
\text { BAPTA- } 1 \text {, } \\
\text { hexapotassium salt } \\
\left(\mathrm{C}_{43} \mathrm{H}_{27} \mathrm{~F}_{2} \mathrm{~K}_{6} \mathrm{~N}_{3} \mathrm{O}_{16}\right)\end{array}$ & Invitrogen O-6806 & 1114.3 & $0.02^{+}$ & $0.02^{+}$ \\
\hline $\mathrm{KOH}$ (1M pH buffer) & Sigma P-1767 & $56.1^{\dagger}$ & $\begin{array}{l}\text { as } \\
\text { required }\end{array}$ & $\begin{array}{l}\text { as } \\
\text { required }\end{array}$ \\
\hline $\mathrm{H}_{2} \mathrm{O}$ & $\begin{array}{l}\text { Elga Water filter } \\
\text { machine }\end{array}$ & 18 & $\begin{array}{l}\text { as } \\
\text { required }\end{array}$ & $\begin{array}{l}\text { as } \\
\text { required }\end{array}$ \\
\hline
\end{tabular}

Notes: *, From $1 \mathrm{M}$ stock solution; ${ }^{+}$, Add to Eppendorf tube prior to calibration procedure; ${ }^{\dagger}$, Larger amount needed for Ca LS due to BAPTA acidity. 
Table 3. Key ions of intracellular $\mathrm{Ca}^{2+}$ calibration solutions

\begin{tabular}{|l|l|l|l|l|}
\hline \multicolumn{1}{|c|}{$\mathrm{Ca}^{2+}(\mathrm{nM})$} & \multicolumn{1}{|c|}{$\mathrm{Mg}^{2+}(\mathrm{mM})$} & ATP $(\mathrm{mM})$ & BAPTA (mM) & \multicolumn{1}{|c|}{$\begin{array}{c}\text { Solution } \\
\text { code }^{\dagger}\end{array}$} \\
\hline 2.39 & 0.49 & 0.62 & 9.79 & $\mathrm{~N} 0$ \\
\hline 26.3 & 0.49 & 0.61 & 8.90 & $\mathrm{~N} 1$ \\
\hline 59.1 & 0.50 & 0.61 & 7.91 & $\mathrm{~N} 2$ \\
\hline 101 & 0.51 & 0.60 & 6.92 & $\mathrm{~N} 3$ \\
\hline 157 & 0.51 & 0.59 & 5.94 & $\mathrm{~N} 4$ \\
\hline 236 & 0.52 & 0.59 & 4.95 & $\mathrm{~N} 5$ \\
\hline 354 & 0.52 & 0.58 & 3.96 & $\mathrm{~N} 6$ \\
\hline 549 & 0.53 & 0.57 & 2.98 & $\mathrm{~N} 7$ \\
\hline 937 & 0.54 & 0.57 & 1.99 & $\mathrm{~N} 8$ \\
\hline 2000 & 0.55 & 0.56 & 1.01 & $\mathrm{~N} 9$ \\
\hline 23000 & 0.58 & 0.52 & 0.1 & $\mathrm{~N} 10$ \\
\hline
\end{tabular}

Note: Nominal free $\mathrm{Ca}^{2+}$ concentration is estimated using webmaxc (Stanford; http://web.stanford.edu/ cpatton/webmaxcS.htm) for $33^{\circ} \mathrm{C}$ following the optimised solution preparation procedure described in Supplementary Fig. 1. 
Table 4. Troubleshooting table

\begin{tabular}{|c|c|c|c|}
\hline Step & Problem & Possible Reason & Possible Solution \\
\hline $\begin{array}{l}\text { Reagent } \\
\text { setup, } \\
\text { Calibration } \\
\text { solution } \\
\text { battery }\end{array}$ & $\begin{array}{l}\text { Logistic } \\
\text { fitting data } \\
\text { points not } \\
\text { perfect }\end{array}$ & $\begin{array}{l}\text { Some of the } \\
\text { calibration steps } \\
\text { were not followed } \\
\text { correctly. }\end{array}$ & $\begin{array}{l}\text { Calibration process } \\
\text { must be repeated } \\
\text { again. }\end{array}$ \\
\hline \multirow[t]{2}{*}{$8 \mathrm{~A} / \mathrm{B}$} & \multirow[t]{2}{*}{$\begin{array}{l}\text { Calcium } \\
\text { level is } \\
\text { rising } \\
\text { steadily }\end{array}$} & $\begin{array}{l}\text { Excessive excitation } \\
\text { laser power }\end{array}$ & $\begin{array}{l}\text { Check that laser power } \\
\text { under the objective } \\
\text { does not exceed } ~ 8 \\
\text { mW. Reduce the laser } \\
\text { power and move to } \\
\text { another ROI or cell. }\end{array}$ \\
\hline & & Poor cell health & $\begin{array}{l}\text { Discard the data and } \\
\text { move to another cell or } \\
\text { ROI }\end{array}$ \\
\hline \multirow[t]{2}{*}{$8 \mathrm{~A} / \mathrm{B}$} & \multirow[t]{2}{*}{$\begin{array}{l}\text { Response } \\
\text { rundown }\end{array}$} & Phototoxic damage & $\begin{array}{l}\text { Reduce laser power if } \\
\text { possible and increase } \\
\text { resting period between } \\
\text { each line scan }\end{array}$ \\
\hline & & Poor cell health & $\begin{array}{l}\text { Discard the data and } \\
\text { move to another cell or } \\
\text { ROI }\end{array}$ \\
\hline \multirow[t]{5}{*}{$8 \mathrm{~A} / \mathrm{B}$} & \multirow{5}{*}{$\begin{array}{l}\text { Not } \\
\text { enough } \\
\text { photons } \\
\text { can be } \\
\text { secured } \\
\text { for a ROI } \\
\text { in an 2D } \\
\text { image }\end{array}$} & Laser power too low & $\begin{array}{l}\text { Check laser power } \\
\text { under the objective, } \\
\text { increase if appropriate. }\end{array}$ \\
\hline & & Wrong filters & $\begin{array}{l}\text { Double check filter } \\
\text { specs and remove } \\
\text { redundant ones. }\end{array}$ \\
\hline & & $\begin{array}{l}\text { Laser alignment } \\
\text { issue }\end{array}$ & $\begin{array}{l}\text { Check optical system } \\
\text { is optimal. }\end{array}$ \\
\hline & & $\begin{array}{l}\text { Fluorophore has } \\
\text { been damaged (e.g. } \\
\text { oxidised) }\end{array}$ & $\begin{array}{l}\text { Make new stock dye } \\
\text { solution to add to a } \\
\text { new aliquot of internal } \\
\text { solution. }\end{array}$ \\
\hline & & $\begin{array}{l}\text { Poor dye loading } \\
\text { procedure }\end{array}$ & $\begin{array}{l}\text { Wait longer to allow } \\
\text { diffusion of dye or } \\
\text { patch a different cell }\end{array}$ \\
\hline
\end{tabular}




\begin{tabular}{|c|c|c|c|}
\hline & & $\begin{array}{l}\text { Chosen ROI is too } \\
\text { deep in the slice }\end{array}$ & $\begin{array}{l}\text { Move to an ROI closer } \\
\text { to slice surface }\end{array}$ \\
\hline & & None of the above & $\begin{array}{l}\text { Consider using line } \\
\text { scan mode or a } \\
\text { smaller ROI. If all fails, } \\
\text { increase OGB-1 } \\
\text { concentration up to } \\
400 \mu \mathrm{M} \text {. }\end{array}$ \\
\hline $8 \mathrm{~A} / \mathrm{B}$ & $\begin{array}{l}\text { Program } \\
\text { reports } \\
\text { insufficient } \\
\text { space }\end{array}$ & $\begin{array}{l}\text { Some file formats } \\
\text { has file size limited }\end{array}$ & $\begin{array}{l}\text { Change the file size } \\
\text { limit in the program } \\
\text { advanced settings }\end{array}$ \\
\hline $10 \mathrm{~A}(\mathrm{i})$ & $\begin{array}{l}\text { Cannot } \\
\text { load FIFO } \\
\text { file into } \\
\text { image }\end{array}$ & $\begin{array}{l}\text { Missing } \\
\text { pixel/line/frame } \\
\text { clock signals }\end{array}$ & $\begin{array}{l}\text { Check that signals are } \\
\text { generated by the laser } \\
\text { scanning microscope } \\
\text { and passed onto the } \\
\text { TCSPC correctly }\end{array}$ \\
\hline $10 \mathrm{~A}$ (iv) & $\begin{array}{l}\text { Insufficient } \\
\text { photons in } \\
\text { binned } \\
\text { pixels or } \\
\text { ROls }\end{array}$ & $\begin{array}{l}\text { ROI structure } \\
\text { volume too small }\end{array}$ & $\begin{array}{l}\text { Increase the } \\
\text { acquisition time, } \\
\text { combine multiple ROIs } \\
\text { of similar types. }\end{array}$ \\
\hline $10 \mathrm{~B}(\mathrm{vi})$ & $\begin{array}{l}\text { Insufficient } \\
\text { photons } \\
\text { result in } \\
\text { large noise } \\
\text { in the } \\
\Delta \mathrm{F} / \mathrm{F}_{0} \\
\text { trace }\end{array}$ & $\begin{array}{l}\text { Similar to step 8B } \\
\text { problems. However, } \\
\text { gated intensity } \\
\text { analysis has much } \\
\text { less stringent } \\
\text { criteria pertinent to } \\
\text { the photon count } \\
\text { rate. }\end{array}$ & $\begin{array}{l}\text { Increase the gating } \\
\text { interval to get less } \\
\text { noisy traces (Fig. } 5 d \text { ). }\end{array}$ \\
\hline
\end{tabular}




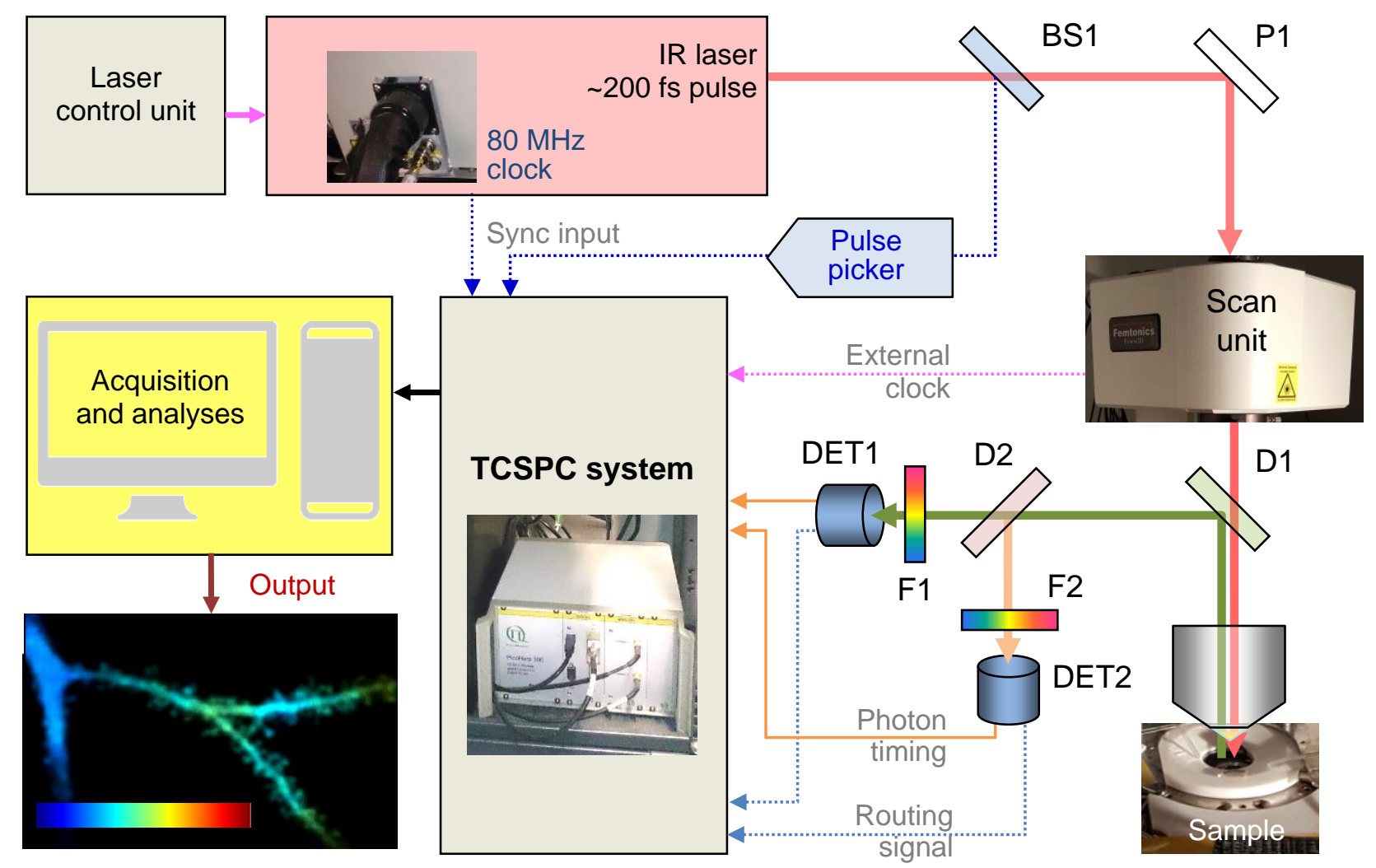

Figure 1 
a

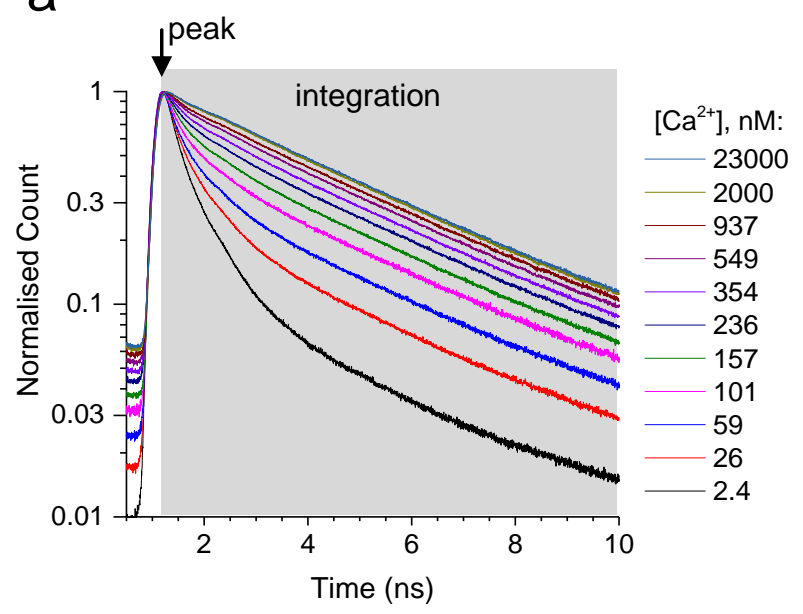

C

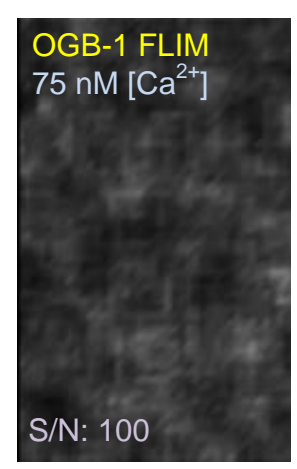

b

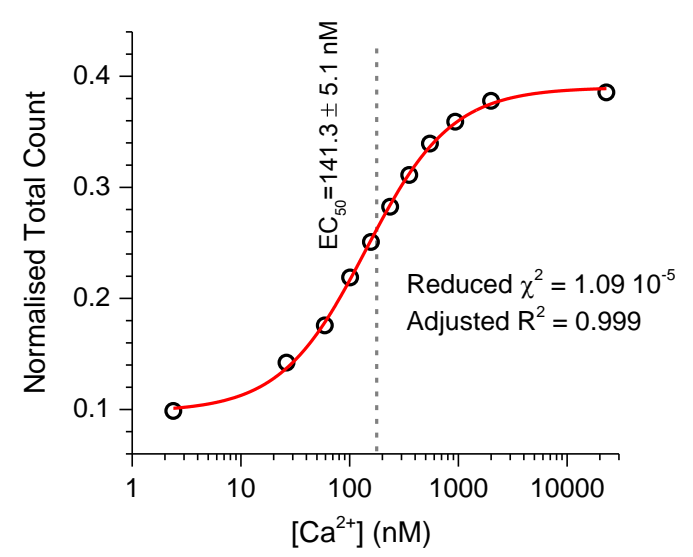

d

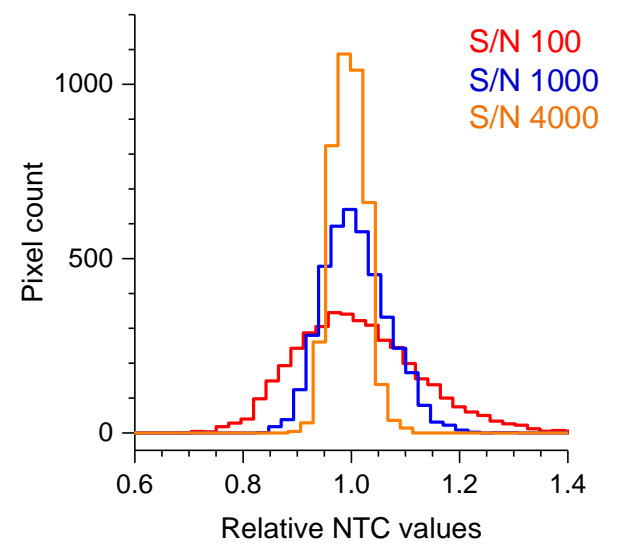

Figure 2 
a
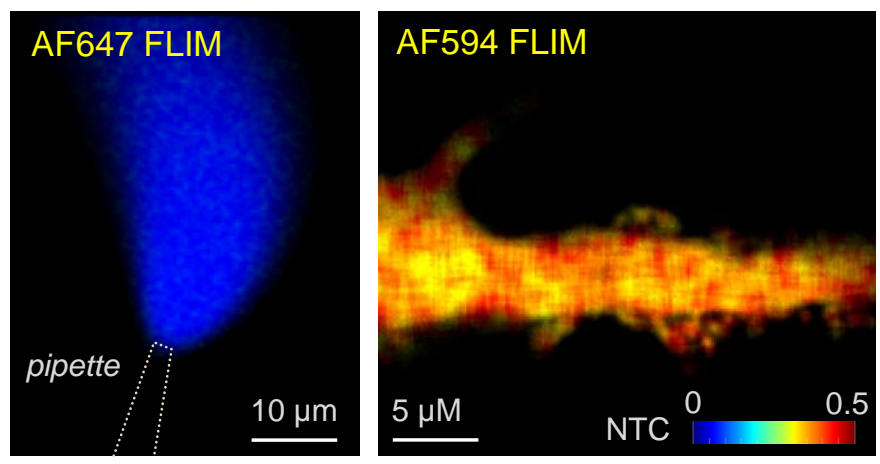

C
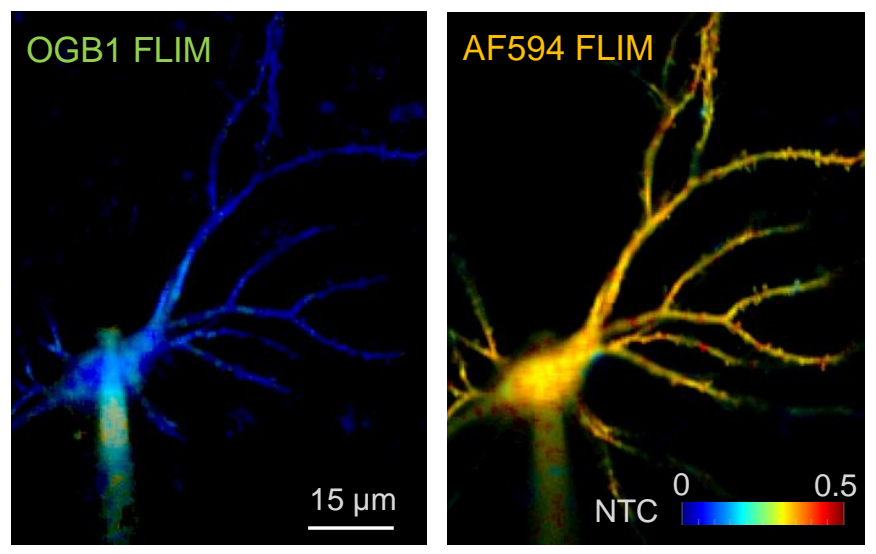

b

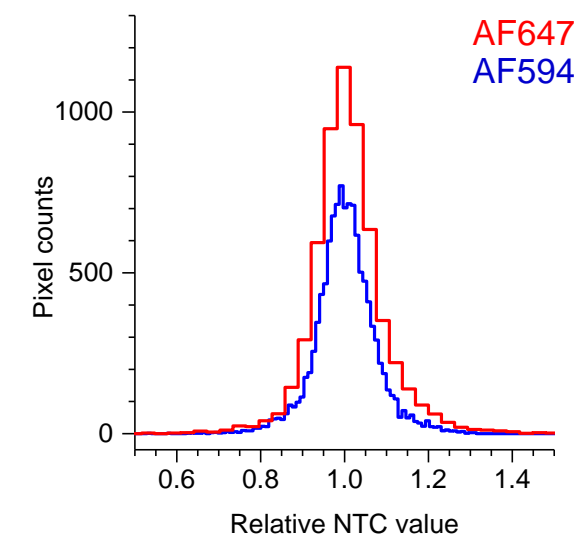

d

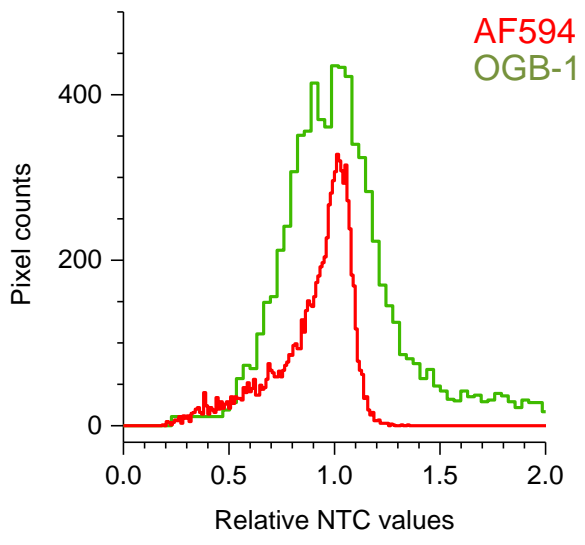

Figure 3 


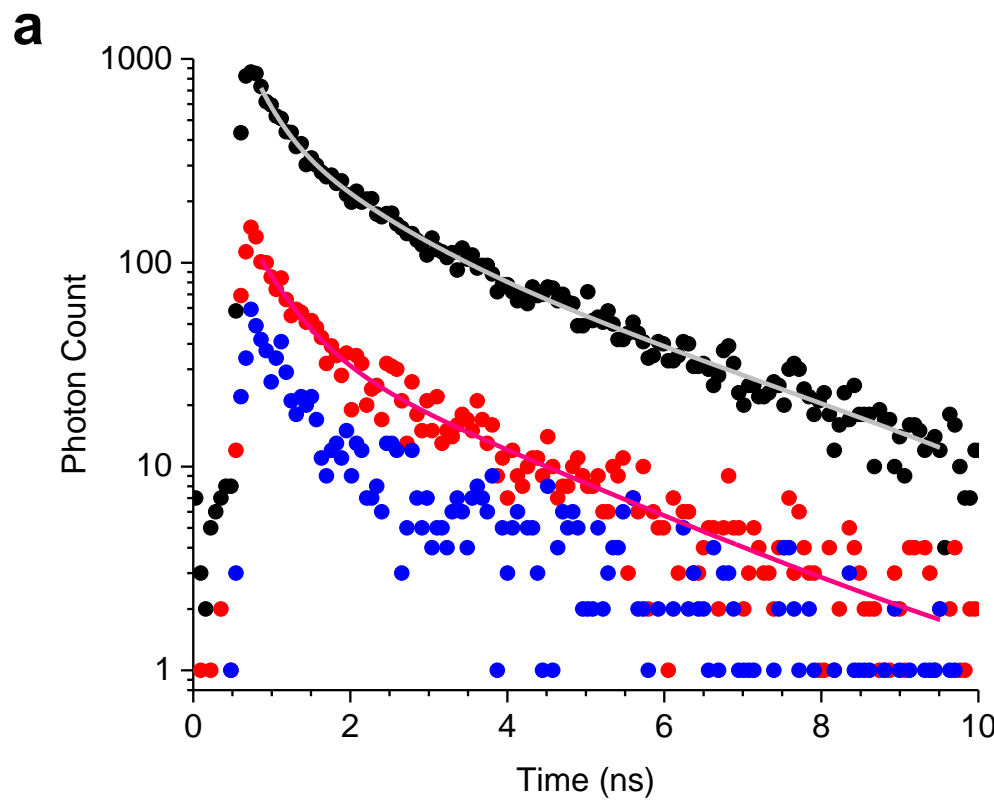

b

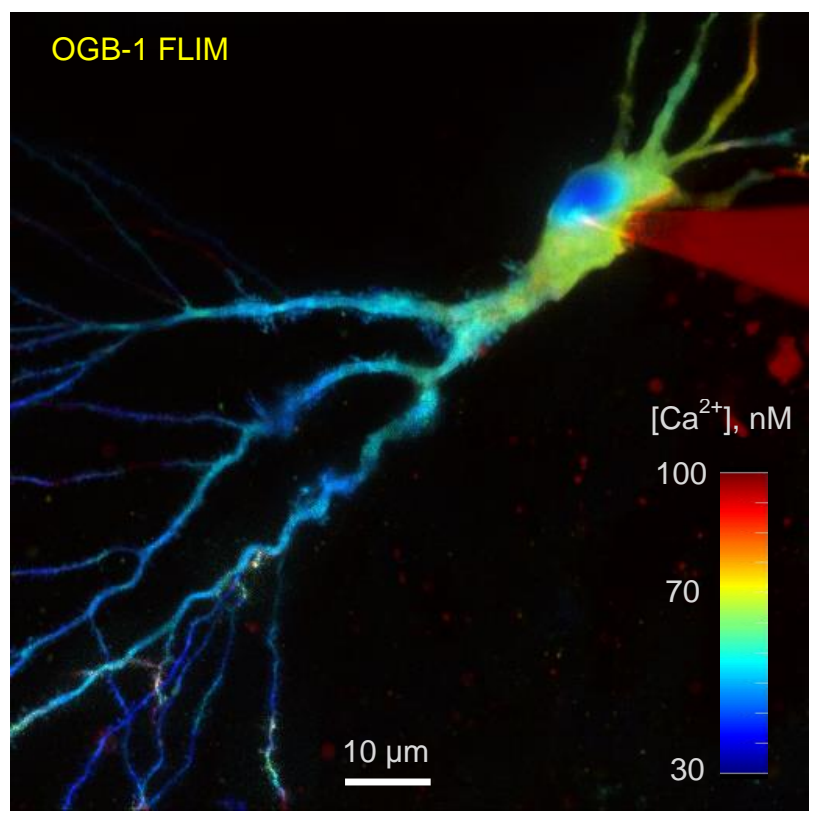

Figure 4 
a

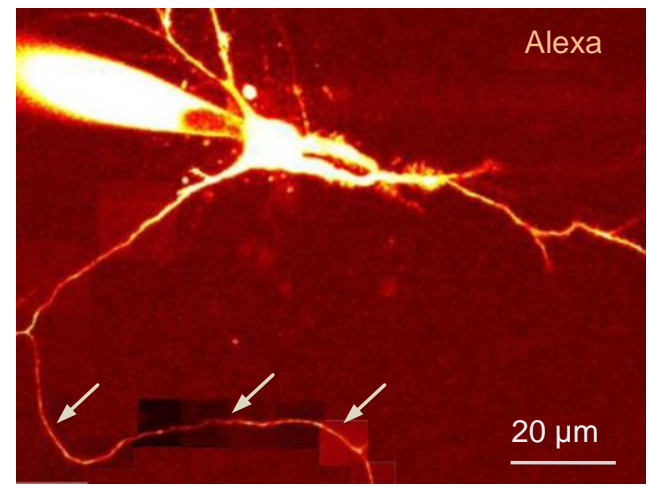

C

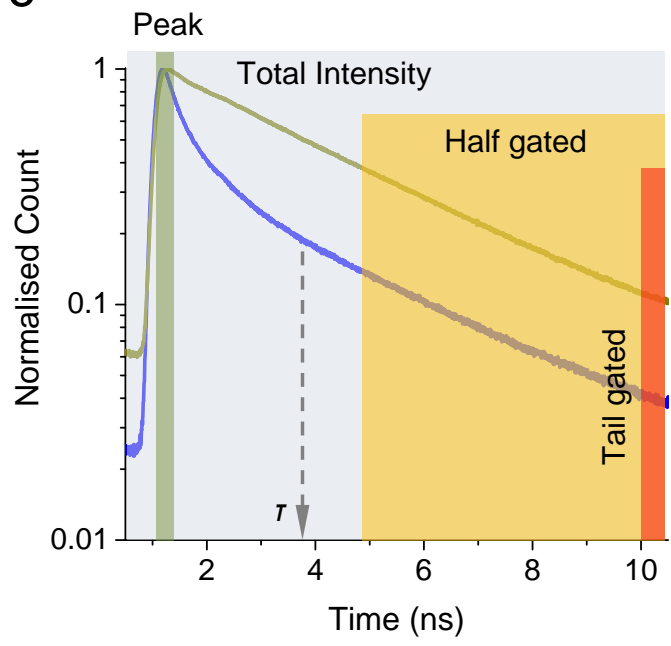

e

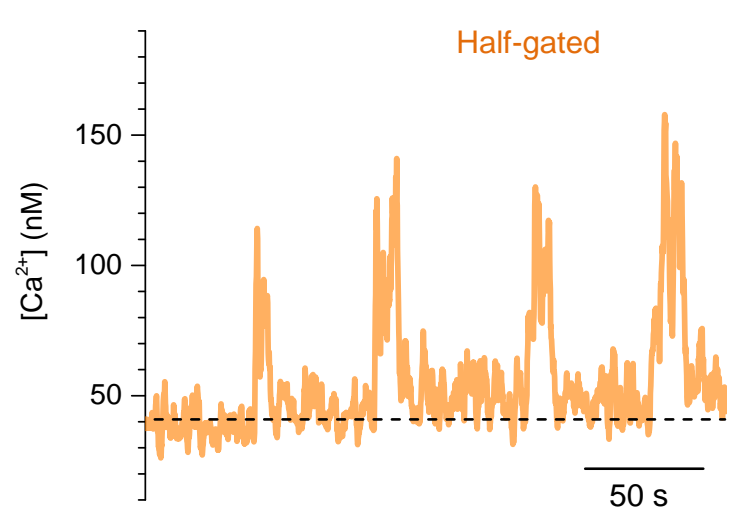

b

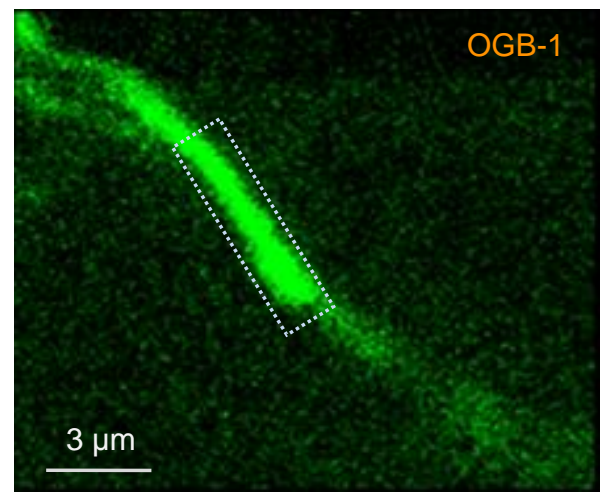

d

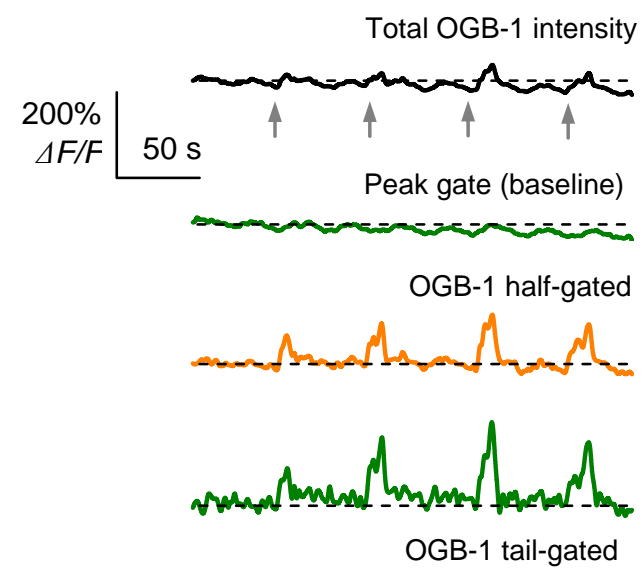

f

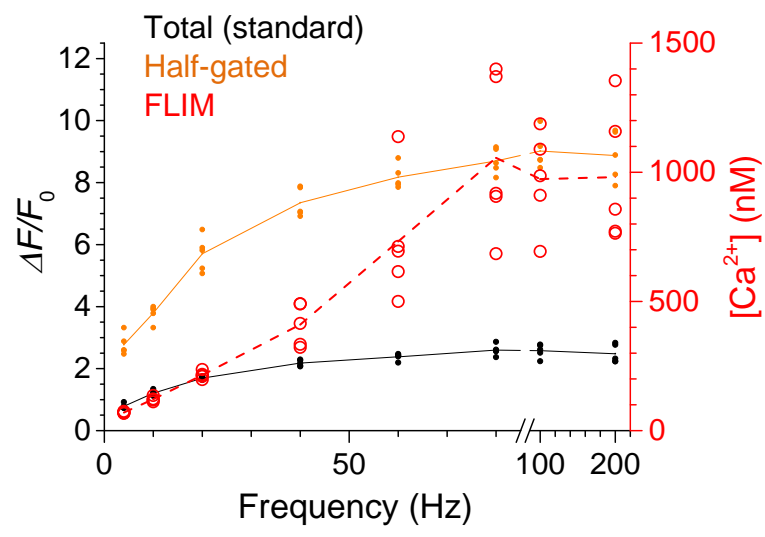

Figure 5 

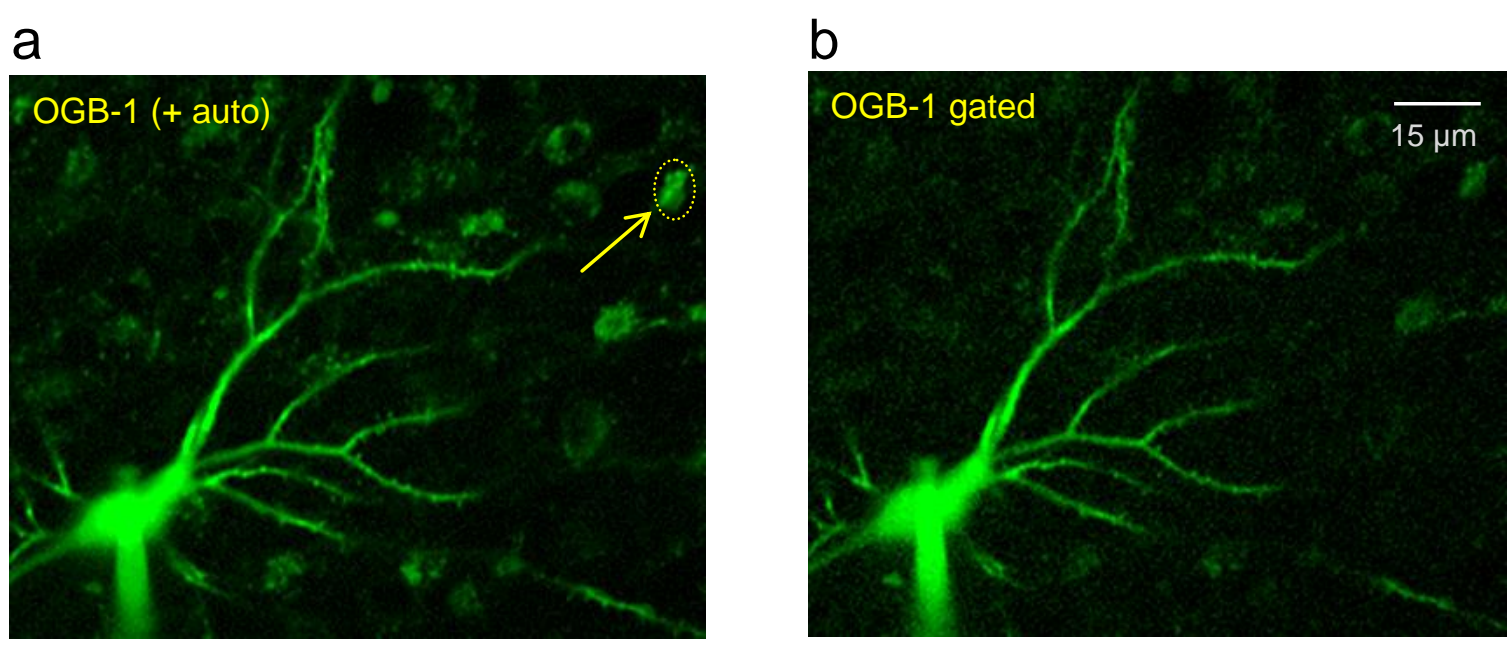

Figure 6 


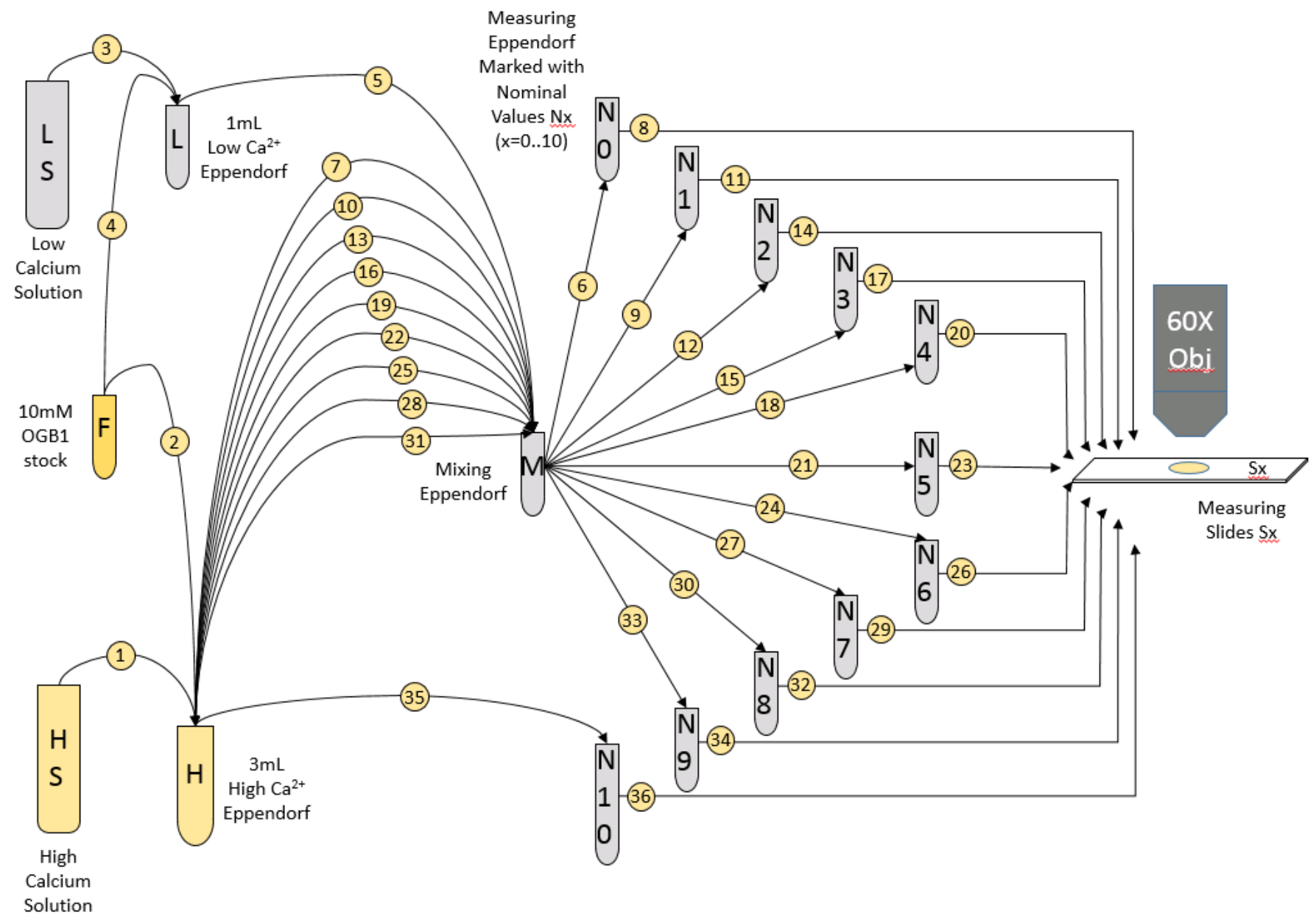

\section{Supplementary Figure 1}

\section{Optimised protocol to prepare calibration solution battery.}

1. Pipette $3 \mathrm{~mL}$ from $\mathbf{H S}$ into the high calcium Eppendorf tube $\mathbf{H}$.

2. Add $6 \mathrm{uL}$ from $10 \mathrm{mM}$ stock OGB-1 solution $\mathbf{F}$ into $\mathbf{H}$ to make up $20 \mathrm{uM}$ concentration of OGB-1 in $\mathbf{H}$. The concentration can be adjusted so that photon count rate is within $1-5 \%$ of the laser pulse repetition rate.

3. Pipette $1 \mathrm{~mL}$ from $\mathbf{L S}$ into the low calcium Eppendorf tube $\mathbf{L}$. 
4. Add $2 u L$ from $\mathbf{F}$ into $\mathbf{L}$ to make up $20 \mathrm{uM}$ concentration of OGB-1 in $\mathbf{L}$. The concentration here should match that of $\mathbf{H}$ as decided at step 2 in order to maintain the same OGB-1 concentration throughout the measurements.

5. Use $\mathbf{L}$ as the mixing Eppendorf $\mathbf{M}$, relabel it from $\mathbf{L}$ to $\mathbf{M}$.

6. Extract $100 \mathrm{uL}$ from $\mathbf{M}$ into a new Eppendorf tube and labelled it $\mathbf{N} \mathbf{0}$ to indicate this is the nominally $0 \mathrm{mM}$ calibration solution.

7. Extract $100 \mathrm{uL}$ from $\mathbf{H}$ and add into $\mathbf{M}$ then mix well. Solution will continue to mix whilst carrying out step 8.

8. Pipette 20uL from No and deposit it onto a clean sterile glass slide labelled S0. Quickly cover it with a coverslip and drop sufficient distilled water on top of the cover slip for water immersion objective. Take care to find the middle of the solution layer using fluorescent intensity. Then take a FLIM trace measurement as outlined in Procedure. This step will be simply stated as taking the measurement hereafter and name of the slides prepared are named as Sx where $\mathrm{x}$ is the nominal calibration values.

9. Extract 111.1uL from $\mathbf{M}$ into a new Eppendorf tube $\mathbf{N} \mathbf{1}$ to indicate this is the nominally $1 \mathrm{mM}$ calibration solution. Extracts from $\mathbf{M}$ are placed into Eppendorf named as $\mathrm{Nx}$ where $\mathrm{x}$ is the nominal calibration values hereafter.

10. Extract 111.1uL from $\mathbf{H}$ and add into $\mathbf{M}$ then mix well.

11. Pipette 20uL from N1 and take measurement $\mathbf{S 1 .}$

12. Extract 125uL from M into N2.

13. Extract $125 \mathrm{uL}$ from $\mathbf{H}$ and add into $\mathbf{M}$ then mix well.

14. Pipette 20uL from N2 and take measurement S2.

15. Extract $142.9 u \mathrm{~L}$ from $\mathbf{M}$ into $\mathbf{N} 3$.

16. Extract $142.9 \mathrm{uL}$ from $\mathbf{H}$ and add into $\mathbf{M}$ then mix well.

17. Pipette 20uL from N3 and take measurement S3.

18. Extract $166.7 \mathrm{uL}$ from $\mathbf{M}$ into $\mathbf{N} 4$. 
19. Extract $166.7 \mathrm{uL}$ from $\mathbf{H}$ and add into $\mathbf{M}$ then mix well.

20. Pipette 20uL from N4 and take measurement S4.

21. Extract 200uL from $\mathbf{M}$ into $\mathbf{N 5}$.

22. Extract $200 \mathrm{uL}$ from $\mathbf{H}$ and add into $\mathbf{M}$ then mix well.

23. Pipette 20uL from N5 and take measurement S5.

24. Extract 250uL from $\mathbf{M}$ into N6.

25. Extract 250uL from $\mathbf{H}$ and add into $\mathbf{M}$ then mix well.

26. Pipette 20uL from N6 and take measurement S6.

27. Extract 333.3uL from $\mathbf{M}$ into $\mathbf{N} 7$.

28. Extract 333.3uL from $\mathbf{H}$ and add into $\mathbf{M}$ then mix well.

29. Pipette 20uL from N7 and take measurement S7.

30. Extract 500uL from $\mathbf{M}$ into N8.

31. Extract $500 \mathrm{uL}$ from $\mathbf{H}$ and add into $\mathbf{M}$ then mix well.

32. Pipette 20uL from N8 and take measurement S8.

33. Take $\mathbf{M}$ and relabel it $\mathbf{N 9 .}$

34. Pipette 20uL from N9 and take measurement S9.

35. Take $\mathbf{H}$ and relabel it $\mathbf{N} 10$.

36. Pipette 20uL from N10 and take measurement $\mathbf{S 1 0 .}$

37. Analyse saved measurements using the NTC analysis in the procedure section (Fig. 2a) to obtain NTC values for each calibration solution.

38. Use smallest estimate of residue calcium (e.g. from all constituent chemicals and glass surfaces) as the nominal $0 \mathrm{mM}$ value, for example $1 \mu \mathrm{M}$ as nominal value. 
39. Use WEBMAXC and key in respective constant parameters such as $\mathrm{pH}$, temperature, [ATP], $\left[\mathrm{Mg}^{2+}\right]$ and [BAPTA] $(10 \mathrm{mM}+$ [OGB-1] used $20 \mu \mathrm{M}$ in this example) as used during calibration procedure to estimate $\left[\mathrm{Ca}^{2+}\right]_{\text {rree. }}$.

40. Alter the $\left[\mathrm{Ca}^{2+}\right]$ from nominal values to estimated values from step 39.

41. Plot NTC value from step $37 \mathrm{vs}$ estimated $\left[\mathrm{Ca}^{2+}\right]_{\text {free }}$ from step 40 , and fit the logistic function $\mathrm{f}(\mathrm{x})=\mathrm{A} 2+(\mathrm{A} 1-\mathrm{A} 2) /\left(1+(\mathrm{x} / \mathrm{x} 0)^{\wedge} \mathrm{p}\right)$ using Levernberg Marquardt algorithm (Fig. 2b). The Adjusted $R^{2}$ value is $>0.99$ and typical reduced $x^{2}$ is in the range of $10^{-5}$. [Troubleshoot]

42. Invert the estimated logistic function to the form $f(x)=x 0^{*}\left((A 1-x) /(x-A 2)^{\wedge}(1 / p)\right.$ with fitted parameter values as the calibration function for calculating all future estimated $\left[\mathrm{Ca}^{2+}\right]_{\text {free }}$ using NTC on images or traces. 


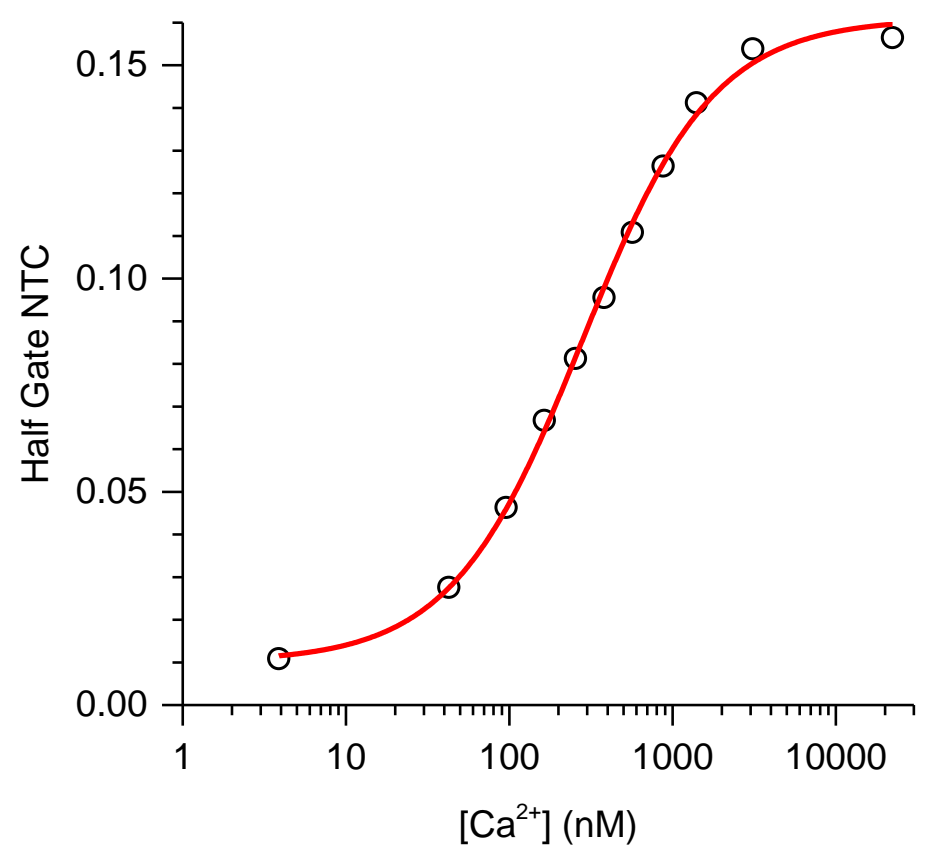

\section{Supplementary Figure 2}

Half-gated NTC calibration for improved monitoring of $\left[\mathrm{Ca}^{2+}\right]$ fluctuations.

Normalised total count values (open circles) obtained by using the integrated count over the 5-10 ns post-pulse interval in the fluorescence decay, normalised against count the peak value (Fig. 5c). Red solid line, best-fit logistic function (as in Fig. 1b); reduced $\mathrm{X}^{2}, 6.5278 \cdot 10^{-6}$; adjusted $\mathrm{R}^{2}, 0.997$. 